\title{
ON THE QUASI-SIMPLE IRREDUCIBLE REPRESENTATIONS OF THE LORENTZ GROUPS
}

\author{
BY \\ ERNEST THIELEKER
}

\begin{abstract}
For $n \geq 2$, let $G(n)$ denote the generalized homogeneous Lorentz group of an $n+1$-dimensional real vector space; that is, $G(n)$ is the identity component of the orthogonal group of a real quadratic form of index $(+,--\ldots-)$. Let $G(n)$ denote a twofold covering group of $G(n)$, and let $S(n) \hat{M}(n)$ be a parabolic subgroup of $G(n)$. We consider the induced representations of $G(n)$, induced by the finite-dimensional irreducible representations of $S(n) \hat{M}(n)$. By an extension of the methods used in a previous paper, we determine precise criteria for the topological irreducibility of these representations. Moreover, in the exceptional cases when these representations fail to be irreducible, we determine the irreducible subrepresentations of these induced representations. By means of some general results of Harish-Chandra together with the main results of this paper, we obtain a complete classification, up to infinitesimal equivalence, of the quasi-simple irreducible representations of the groups $G(n)$.
\end{abstract}

1. Introduction. Let $n$ be an integer equal to or greater than 2, and let $G(n)$ be the identity component of the orthogonal group of a real quadratic form of signature $(+,--\ldots-)$. Let $\hat{G}(n)$ denote a two-fold covering group of $G(n)$. For $n \geq 3, \hat{G}(n)$ is simply connected. Let $\hat{K}(n)$ be a maximal compact subgroup of $\hat{G}(n)$ and let $\hat{S}(n) \hat{M}(n)$ be a parabolic subgroup of $\hat{G}(n)$. See the next section for a precise definition of these subgroups for the situation under study. We consider the induced representations of $\hat{G}(n)$ induced, in the sense of Bruhat [4], by the finite-dimensional irreducible representations of the subgroup $\hat{S}(n) \hat{M}(n)$. We determine precisely for what representations of $\hat{S}(n) \hat{M}(n)$ these induced representations are irreducible, and in the nonirreducible cases, we determine the irreducible subrepresentations. We remark that all the finite-dimensional representations, as well as certain "discrete" series of representations occur as subrepresentations of these induced representations, in the nonirreducible cases. In case $n=2, \hat{G}(2)$ is isomorphic to $\operatorname{SL}(2, \mathbf{R})$. For this case, it is known that the finite-dimensional representations occur as subrepresentations of these induced representations for certain "integral" values of the character of $\hat{S}(n) \hat{M}(n)$. When

Received by the editors August 24, 1971 and, in revised form, March 1, 1972.

AMS (MOS) subject classifications (1970). Primary 22E45, 22D12, 22E43; Secondary 22D10, 22D30, 43A65.

Key words and phrases. Quasi-simple representations, topologically completely irreducible representations, irreducibility criteria for induced representations, classification of quasi-simple representations, representations of real semisimple Lie groups, representations of generalized homogeneous Lorentz groups, representations of Lorentz groups, representations of the deSitter group, nonunitary representations on a Banach space, unitary representations of generalized homogeneous Lorentz groups, infinitesimal equivalence, Naimark equivalence, modules over universal enveloping algebras. 
the induced representations contain such a finite-dimensional subrepresentation, there is a supplementary subspace that is invariant under another induced representation related to the original one by a Weyl reflection of the character of $\hat{S}(n) \hat{M}(n)$. The latter representation is essentially a direct sum of two discrete series of representations. There is another way of describing the relationship between the discrete series and finite-dimensional representations in the case of $\hat{G}(2)$, namely the finite-dimensional representation may be regarded as a quotient representation of an induced representation modulo a discrete-series-representation. The latter may be regarded as a quotient representation of an induced representation modulo a finite-dimensional one. This relationship between subrepresentations and quotient representations generalizes in a manner that is made precise in $\$ \S 10$ and 11 . This relationship enables us to apply some results of Harish-Chandra to obtain a complete classification of the irreducible quasisimple representations of $\hat{G}(n)$ up to infinitesimal equivalence. The main results of the paper are summarized in Theorem 6 . Of course, no claim is made that the various discrete series that occur in this analysis are unitarizable, although in case $n=2$, the discrete series is in fact unitarizable, as are some of the discrete series for even $n$, according to results of Dixmier [6] and Takahashi [17]. Results similar to some of those of the present paper have been announced by Hirai [11 a,b,c]. Hirai has also announced results on the characters of the irreducible representations of these groups.

The methods used in this paper are an extension of those used in [16] for the case of semidirect product groups. We use a basic result of Harish-Chandra according to which the topological irreducibility of a $\hat{K}(n)$-finite representation of $\hat{G}(n)$ is equivalent to the algebraic irreducibility of the corresponding $U(\mathbf{G}(n))$ module of $R(n)$-finite vectors, where $U(\mathbf{G}(n))$ denotes the universal enveloping algebra of the Lie algebra of $\hat{G}(n)$. We use some of the methods of [18] to reduce the problem of showing irreducibility of these $U(\mathbf{G}(n))$-modules to the problem of showing that these $U(\mathbf{G}(n))$-modules are modules over a certain commutative algebra. In case the induced representations are irreducible, the commutative algebra used is a polynomial algebra defined on a Cartan subspace of the Lie algebra $\mathbf{G}(n)$. For the Lorentz groups, this algebra is essentially the pointwise algebra generated by the spherical functions defined on the $n-1$-sphere, and is analogous to the one used in [18]. For the case when the induced representations are not irreducible, this algebra must be modified. The connection between the $U(\mathbf{G}(n))$-module structure of the space of $\hat{K}(n)$-finite vectors and the module structure of this space over the algebra of spherical harmonics is made by Lemma 1 and Theorem 1, the main computational theorem of the paper.

We turn to an explanation of the notation and basic definitions used in this paper. If $L$ is a real Lie group, we denote by $\mathbf{L}$ its $L$ ie algebra, and by $\mathbf{L}_{\mathbf{c}}$ the complexification of $\mathbf{L}$. We denote the complex universal enveloping algebra of $\mathbf{L}$ by $U(\mathbf{L})$. If $K$ is a Lie subalgebra of $L$, then $U(\mathbf{K})$ is identified as the subalgebra 
of $U(\mathbf{L})$ generated by $\mathbf{C}$ and $\mathbf{L}$. If $K$ is a compact group, we denote by $\Omega(K)$ the set of equivalence classes of irreducible unitary representations of $K$, or equivalently, the set of equivalence classes of irreducible finite-dimensional representations of $K$. If $V$ is a vector space, and $g \rightarrow \Pi(g)$ is a representation of a group $G$ on $V$, we shall express this information by the symbol $[V, \Pi]$. If $\mathbf{G}$ is a Lie algebra and $d \Pi$ is a module action of $\mathbf{G}$ on $V$, we shall also use the notation $[V, d \Pi]$ to denote the G-module $V$, together with this action. We also use the same symbol to denote the natural extension of this module to $U(\mathbf{G})$.

Let $G$ be a real Lie group, and let $K$ be a compact subgroup of $G$. Let $\left[H_{\Pi}, \Pi\right]$ be a continuous representation of $G$ on a Banach space $H_{\Pi}$. If $V$ is a linear subspace of $H_{\Pi}$ consisting of infinitely differentiable vectors, then $[V, d \Pi]$ shall denote the correponding $U(\mathbf{G})$-module. Following Harish-Chandra, we call the representation $\left[H_{\Pi}, \Pi\right]$ quasi-simple, if $\left[H_{\Pi}^{\infty}, d \Pi\right]$ restricts to scalar multiplication on the center of $U(\mathbf{G})$, where $H_{\Pi}^{\infty}$ is the linear subspace of $H_{\Pi}$ consisting of infinitely differentiable vectors. Following Godement [8] the representation is called completely irreducible if the strongly closed algebra generated by the set operators $\{\Pi(g): g \in G\}$ coincides with the algebra of all bounded operators on $H_{\Pi}$. We employ the terminology of [18] regarding $K$-finite vectors in $H_{\Pi}, K$-finite representations, and infinitesimal equivalence of $K$-finite representations. If $G$ is connected and a semisimple matrix group, as in the case with the groups under consideration, and if $K$ is a maximal compact subgroup of $G$, then it is known that every completely irreducible representation of $G$ is $K$-finite. (See [8].) It is also known that every quasi-simple representation of $G$ is $K$-finite [9a, Theorem 1]. A stronger result is also known (see $\S 11$ ). Let us assume that $\left[H_{\Pi}, \Pi\right]$ is $K$ finite. We say that $\left[H_{\Pi}, \Pi\right]$ is unitarizable if it is infinitesimally equivalent to a unitary representation. If $\left[H_{\Pi}, \Pi\right]$ is unitarizable, then this unitary representation is unique up to unitary equivalence [9a, Theorem 8]. An extension of HarishChandra's argument by Fell [7] shows that infinitesimal equivalence of $K$-finite irreducible representations is equivalent to Naimark equivalence of completely irreducible representations. If $\left[H_{\Pi}, \Pi\right]$ is a $K$-finite representation of $G$, we denote by $d H_{\Pi}$ the dense linear subspace of $K$-finite vectors. Of course, for finitedimensional representations we have the equality $H_{\Pi}=d H_{\Pi}$.

We now outline the paper. In $\S 2$ the induced representations induced by a parabolic subgroup of $G$ are discussed. $\$ 3$ contains the main computational lemma of the paper. This lemma is derived in the context of a general split-rank one simple Lie group. The lemma replaces, in part, Lemma 1 of [18] for the semidirect product case. The lemma of $\S 3$ is improved upon in $\$ 4$ with the aid of some known information, in the case of the Lorentz groups, on the tensor products of $\mathbf{K}(n)$-modules, and the eigenvalues of the Casimir operators in these modules. For general facts on semisimple Lie groups and Lie algebras quoted in $\S 3$, the reader is referred to [10]. The basic properties of $\operatorname{Spin}(1, n)$ used in this paper may be derived by methods similar to those of [5, p. 64]. The first application of our methods appears in $\S 6$, where sufficient conditions for 
irreducibility are obtained. These conditions turn out to be "almost" necessary as well; however, it is more convenient to sort out the complete state of affairs in Theorem $6(\$ 11)$. In $\$ 7$ the commutative algebra used in the proof of Theorem 2 is modified to handle the nonirreducible situation. Here, for the first time, explicit use is made of the fact that $\hat{K}(n)$-irreducible subrepresentations occur with multiplicity no greater than one, in the induced representations of $\hat{G}(n)$. The theorems proved in $\$ 8$, together with Theorem 2 , then exhaust all the possibilities for irreducible subrepresentations of the induced representations. The theorem in $\S 11$ summarizes the results of this paper and includes criteria for the infinitesimal equivalence of the induced representations, in the irreducible cases, as well as the structure of the quasi-simple representations. Some general results of HarishChandra are used to obtain this information. Along the way one obtains the infinitesimal equivalence between certain subrepresentations and certain quotient representations. $\$ 12$ contains some comparisons between our results and some known results for the generalized Lorentz groups. For the purposes of these comparisons, the computation of the eigenvalues of the Casimir operator, undertaken in $\S 9$, is helpful.

2. The infinitesimal equivalence classes of induced representations. Let $G$ be a semisimple Lie group which has a faithful matrix representation. Let $K$ be a maximal compact subgroup of $G$, and let $\mathbf{G}=\mathbf{K} \oplus \mathbf{P}$ be the Cartan decomposition of the Lie algebra $\mathbf{G}$, corresponding to the maximal compact subalgebra $K$. Let $\boldsymbol{\theta}$ be the Cartan involution corresponding to this decomposition. Pick a maximal abelian subalgebra $\mathbf{A}$ of $\mathbf{P}$, and fix a lexicographical ordering in the real dual of $\mathbf{A}$. Let $\mathbf{N}$ denote the internal direct sum of the eigenspaces of $\operatorname{ad}(\mathbf{A})$, corresponding to the roots which are positive in this ordering. Then $\mathbf{N}$ is a maximal nilpotent subalgebra of $\mathbf{G}$, and $\mathbf{S}=\mathbf{N} \oplus \mathbf{A}$ is a maximal solvable subalgebra of $\mathbf{G}$. Let $S, A$, and $N$ denote the analytic subgroups of $G$ corresponding to the subalgebras $\mathbf{S}, \mathbf{A}$, and $\mathbf{N}$, respectively. Let $M$ denote the centralizer of $\mathrm{A}$ in $K$, under the adjoint representation. Then $G=S K$ is an Iwasawa decomposition of $G$, with $S$ a closed solvable subgroup of $G$ homeomorphic to a Euclidean space, and $S \cap K=\{1\}$. The subset $S M$ is a minimal parabolic subgroup of $G$, that is, the normalizer of $S$ in $G$, and $M=S M \cap K$.

Let $H$ be a finite-dimensional Hilbert space equipped with an inner product ( , ). Suppose $[\underline{H}, \mu]$ is an irreducible $M$-module, and let $\Lambda$ be a complex character of the subgroup $S$. Since $N$ lies in the commutator subgroup of $S$, it follows that $\Lambda(N)=1$. The infinitesimal character, $d \Lambda$, is a linear form on $\mathbf{S}$ which is zero on $\mathbf{N}$. In the real-rank one case, in which the generalized homogeneous Lorentz groups fall, $d \Lambda$ is determined by a single complex number $\lambda$, its value on a nonzero element $H \in \mathbf{A}$. Since $M$ is in the normalizer of $S$, and since $M$ fixes the elements of $A$, it follows that, for all $s \in S$, and $m \in M, \Lambda\left(m s m^{-1}\right)=\Lambda(s)$. It follows that one may define a representation $\Lambda \mu$ of the parabolic subgroup $S M$ by means of the formula: $\Lambda \mu(s m)=\Lambda(s) \mu(m)$, for all $s \in S$, and $m \in M$. 
Let $C_{\Lambda \mu}(G, \underline{H})$ denote the linear space consisting of functions $F$ from $G$ to $\underline{H}$ which satisfy the subsidiary condition:

$$
F(p g)=\Lambda \mu(p) F(g)
$$

for $p \in M S$, and $g \in G$. We note that each function in the space $C_{\Lambda \mu}(G, \underline{H})$ is uniquely determined by its restriction to the compact subgroup $K$. Moreover, let $C_{\mu}(K, \underline{H})$ denote the linear space consisting of such restrictions. Then $C_{\mu}(K, \underline{H})$ consists of the space of all $\underline{H}$-valued continuous functions defined on $K$ and which satisfy the condition:

$$
\psi(m k)=\mu(m) \psi(k)
$$

for all $m \in M, k \in K$, and $\psi \in C_{\mu}(K, \underline{H})$. In fact, if $\psi$ is a continuous function from $K$ to $\underline{H}$ which satisfies condition (2), then we define a function $F$ from $G$ to $H$ which satisfies condition (1) as follows. For $g \in G$, let $F(g)=\Lambda(\sigma(g)) \psi(\kappa(g))$, where $\sigma(g)$ and $\kappa(g)$ are the uniquely defined elements in $S$ and $K$, respectively, defined by the Iwasawa decomposition of the element $g$. It is known that the functions $g \rightarrow \sigma(g)$ and $g \rightarrow \kappa(g)$ are real-analytic functions from $G$ to $S$ and $G$ to $K$, respectively. It follows that $F$ is continuous. Moreover, if $\psi$ is real-analytic, it follows that that $F$ is analytic. The fact that $F$ satisfies condition (1) is seen from the following observations: $\sigma(m g)=m \sigma(g) m^{-1}, \kappa(m g)=m \kappa(g)$ and $\Lambda(\sigma(m g))=\Lambda\left(m \sigma(g) m^{-1}\right)$, for $m \in M$, and $g \in G$. Thus the assertion is established.

It is obvious that the restriction-to- $K$ map which takes the space $C_{\Lambda \mu}(G, \underline{H})$ onto the space $C_{\mu}(K, \underline{H})$ is a $K$-module isomorphism for the action $R$ of right translations of functions.

We turn the space $C_{\Lambda \mu}(G, \underline{H})$ into a Banach space by equipping it with the "sup" norm:

$$
\|F\|_{\infty}=\sup _{k \in K}(F(k), F(k))^{1 / 2},
$$

for $F \in C_{\Lambda \mu}(G, \underline{H})$. This defines a norm on $C_{\mu}(K, \underline{H})$ as well.

We may also define an inner product on these spaces by means of the formula:

$$
\langle F, G\rangle=\int_{K}(F(k), G(k)) d k,
$$

where the integral is the Haar integral over $K$, and $F$ and $G$ are elements of $C_{\Lambda \mu}(G, \underline{H})$. Let $L_{\Lambda \mu}^{2}(G, \underline{H})$ denote the completion of $C_{\Lambda \mu}(G, \underline{H})$ with respect to the Hilbert space norm defined by this inner product.

Let $P$ denote the positive square root of the determinant of the adjoint representation of $S$. Then $d P$ is the linear form on $\mathbf{S}$ which is zero on $\mathbf{N}$ and equal to one half the sum of the positive roots on $\dot{A}$. Then, for any character $\Lambda$ on $S$, $\Lambda P$ is also a character of $S$. The induced representation of $G$, induced by $\Lambda \mu$, is, by definition, the representation: $\left[L_{\Lambda P \mu}^{2}(G, \underline{H}), R\right]$, and the continuous induced repre- 
sentation of $G$, induced by $\Lambda \mu$, is by definition, the representation $\left[C_{\Lambda P \mu}(G, \underline{H}), R\right]$. It is a known fact that the action $R$ is continuous for both of these Banach spaces. (See [4].)

For a given representation $[\underline{H, \mu]}$ of the subgroup $S M$, both of these representations are $K$-finite, and infinitesimally equivalent. In fact, let $[\omega] \in \Omega(K)$, and let $\chi_{\omega}$ be the irreducible character of $K$, normalized in such a manner that it is an idempotent under convolution: $\chi_{\omega} * \chi_{\omega}=\chi_{\omega}$. Then the operator $E_{\omega}$, defined on $L_{\Lambda \mu}^{2}(G, \underline{H})$ by the formula

$$
E_{\omega} F=\int_{K} \chi_{\omega}(k) R(k) F d k,
$$

with $F \in L_{\Lambda \mu}^{2}(G, \underline{H})$, is an Hermitian projection on $L_{\Lambda \mu}^{2}(G, \underline{H})$. Its range is the $K$ isotypic subspace corresponding to the representation class $[\omega]$. We note moreover, that the range of this projection is contained in the linear subspace $C_{\Lambda \mu}(G, \underline{H})$. Even more, this range is finite dimensional and consists of realanalytic functions. Thus, it follows from the Peter-Weyl theorem that the representations $\left[L_{\Lambda \mu}^{2}(G, \underline{H}), R\right]$ and $\left[C_{\Lambda \mu}(G, \underline{H}), R\right]$ have a common dense $U(G)$ module of $K$-finite vectors, and are thus infinitesimally equivalent. This $U(\mathbf{G})$ module is given by the (algebraic) direct sum

$$
d C_{\Lambda \mu}(G, \underline{H})=\operatorname{span}_{\mathbf{C}}\left\{E_{\omega} L_{\Lambda \mu}^{2}(G, \underline{H}):[\omega] \in \Omega(K)\right\} .
$$

By some abuse of notation, we shall use the symbol $E_{\omega}$ to denote the projection onto the $k$-isotypic subspace belonging to the class [ $\omega$ ], defined for any $K$-module consisting of square-integrable functions on $K$. In particular, we shall have occasion to consider the slightly more general spaces $L_{\Lambda}^{2}(G, \underline{H})$, and $C_{\Lambda}^{\infty}(G, \underline{H})$. The latter are defined, respectively, as the set of all square-integrable functions, and the set of all $C^{\infty}$-functions which satisfy the condition $f(s g)=\Lambda(s) f(g)$, and which have range in $H$. It is easy to verify that these spaces are linearly equivalent to the tensor products $L_{\Lambda}^{2}(G) \otimes \underline{H}$ and $C_{\Lambda}^{\infty}(G) \otimes \underline{H}$, respectively. For any representation $\mu$ of $M, d C_{\Lambda \mu}(G, \underline{H})$ is a linear subspace of $C_{\Lambda}^{\infty}(G, \underline{H})$.

3. A basic lemma. The main result of this section relates the action of the Lie algebra $\mathbf{G}$ to the action of pointwise multiplication by certain polynomial functions on the homogeneous space $M \backslash K$. The result, Lemma 1 , is analogous to, but more complicated than, Lemma 1 in [18]. The first step in the argument applies to any semisimple real Lie group.

Let $d R$ and $d L$ be the differentials of the right regular representation and the left regular antirepresentation, respectively, defined on $C^{\infty}(G)$. These actions may be defined by $d R(X) f(g)=\left.(d / d t)(f(g \exp t X))\right|_{t=0}$, and $d L(X)$ $\left.\cdot(f(g \exp t X))\right|_{t=0}$, for all $X \in \mathbf{G}, f \in C^{\infty}(G)$, and $g \in G$.

Let $B$ be the Killing form on $G$. We define a positive definite form on $\mathbf{G}$ by $(X, Y)=-c B(X, \theta Y)$, for $X, Y \in \mathbf{G}$, where $c$ is a suitable number. Now let $\left\{Z_{1}, \ldots, Z_{r}\right\}$ be an orthonormal basis of $\mathbf{N}$, with respect to the Euclidean inner 
product ( , ). Let $\left\{H_{1}, \ldots, H_{\ell}\right\}$ be an orthonormal basis of $\mathbf{A}$, and let $\left\{U_{1}, \ldots, U_{s}\right\}$ be an orthonormal basis of $\mathbf{M}$. For each index $i$, with $1 \leq i \leq r$, let $X_{i}$ and $Y_{i}$ be defined by the formulas:

$$
Y_{i}=2^{-1 / 2}\left(Z_{i}-\theta Z_{i}\right), \quad X_{i}=2^{-1 / 2}\left(Z_{i}+\theta Z_{i}\right) .
$$

Then $\left\{H_{1}, \ldots, H_{l}, Y_{1}, \ldots, Y_{r}\right\}$, and $\left\{X_{1}, \ldots, X_{r}, U_{1}, \ldots, U_{s}\right\}$ are orthonormal bases of $\mathbf{P}$ and $\mathbf{K}$ respectively.

Now, let $F \in C_{\Lambda}^{\infty}(G)$, for $\Lambda$ a complex character on $S$. Then, for all $Y \in \mathbf{P}$, and $k \in K$, we have

$$
d R(Y) f(k)=\left.(d / d t) f(k \exp t Y)\right|_{t=0}=\left.(d / d t) f(\exp (t \operatorname{Ad}(k) Y) k)\right|_{t=0} .
$$

Let $\rho$ denote the restriction of the action $\operatorname{Ad}$ of $K$ on $\mathbf{P}$. Then we have

$$
\operatorname{Ad}(k) Y=\sum_{i=1}^{l}\left(\rho(k) Y, H_{i}\right) H_{i}+\sum_{i=1}^{r}\left(\rho(k) Y, Y_{i}\right) Y_{i}
$$

From (3) we have $Y_{i}+X_{i} \in \mathbf{N}$. Hence, $d L\left(Y_{i}\right) f(k)=-d L\left(X_{i}\right) f(k)$. Thus we have

$$
(d R(Y) f)(k)=\sum_{i=1}^{l} d \Lambda\left(H_{i}\right)\left(\rho(k) Y, H_{i}\right) f(k)-\sum_{i=1}^{r}\left(\rho(k) Y, Y_{i}\right)\left(d L\left(X_{i}\right) f\right)(k) .
$$

Now assume that $G$ has split rank 1 . Hence, $\ell=1$. The set of restricted positive roots, in this case, is $\{\alpha\}$, or $\{\alpha, 2 \alpha\}$. Let $H \in \mathbf{A}$, such that $\alpha(H)=1$, and $(H, H)=1$. The last condition determines the number $c$. Let $\mathbf{N}_{1}$ and $\mathbf{N}_{2}$ be defined as the eigenspaces $\operatorname{ad}(H)$ in $\mathbf{G}$, belonging to the eigenvalues 1 and 2 , respectively. Then $\mathbf{N}=\mathbf{N}_{1} \oplus \mathbf{N}_{2}$. Hence, we may and do choose the basis $\left\{Z_{1}, \ldots, Z_{r}\right\}$ such that $\left\{Z_{1}, \ldots, Z_{r_{1}}\right\}$ is a basis of $N_{1}$, and $\left\{Z_{r_{1}+1}, \ldots, Z_{r}\right\}$ is a basis of $\mathbf{N}_{2}$. Let $\Omega_{K}$ be the Casimir operator of $\mathbf{K}$ given by $\Omega_{K}=-\sum_{i=1}^{r} X_{i}^{2}-\sum_{i=1}^{s} U_{i}^{2}$. Then we have the following lemma.

Lemma 1. If $G$ has split rank 1 , then for all $Y \in \mathbf{P}$, and $f \in C_{\Lambda}^{\infty}(G)$, the function $d R(Y) f$ is an element of $C_{\Lambda}^{\infty}(G)$ defined, for all $k \in K$, by

$$
\begin{aligned}
(d R(Y) f)(k)= & \left(\lambda-\frac{1}{2} \mu\right)(\rho(k) Y, H) f(k)+\frac{1}{2} d R\left(\Omega_{K}\right)((\rho(k) Y, H) f(k)) \\
& -\frac{1}{2}(\rho(k) Y, H) d R\left(\Omega_{K}\right) f(k) \\
& +\frac{1}{2} \sum_{i=r_{1}+1}^{r} d L\left(X_{i}\right)(\rho(k) Y, H) d L\left(X_{i}\right) f(k),
\end{aligned}
$$

where $\lambda=d \Lambda(H)$, and $\mu$ is the eigenvalue of $\Omega_{K}$ in the irreducible representation $[P, d \rho]$ of $K$. This eigenvalue is a positive number.

Proof. Since $\Omega_{K}$ is in the center of $U(\mathbf{K})$, we have $\left(d L\left(\Omega_{K}\right) f\right)(k)=\left(d R\left(\Omega_{K}\right) f\right)(k)$, for all $k \in K$, and $f \in C_{\Lambda}^{\infty}(G)$. By the Leibnitz rule, applied to the derivations $d L\left(X_{i}\right)$, and $d L\left(U_{i}\right)$, and the fact that $\left[U_{i}, H\right]=0$, we have for all $Y \in \mathbf{P}$, and $k \in K$, 


$$
d R\left(\Omega_{K}\right)((\rho(k) Y, H) f(k))=f(k) d R\left(\Omega_{K}\right)(\rho(k) Y, H)+(\rho(k) Y, H) d R\left(\Omega_{K}\right) f(k)
$$

$$
-2 \sum_{i=1}^{r}\left(d L\left(X_{i}\right)(\rho(k) Y, H)\right)\left(d L\left(X_{i}\right) f(k)\right) .
$$

Now, by (3),

$$
\begin{aligned}
{\left[H, X_{i}\right] } & =2^{-1 / 2}\left[H, Z_{i}+\theta Z_{i}\right]=Y_{i}, & & \text { for } 1 \leq i \leq r_{1}, \\
& =2 Y_{i}, & & \text { for } r_{1}+1 \leq i \leq r .
\end{aligned}
$$

Hence, the last term of $(*)$ becomes

$$
-2 \sum_{i=1}^{r_{1}}\left(\rho(k) Y, Y_{i}\right) d L\left(X_{i}\right) f(k)-4 \sum_{i=r_{1}+1}^{r}\left(\rho(k) Y, Y_{i}\right) d L\left(X_{i}\right) f(k) .
$$

By (4), the latter expression is

$$
2(d R(Y) f)(k)-2 \lambda(\rho(k) Y, H) f(k)-\sum_{i=r_{1}+1}^{r} d L\left(X_{i}\right)(\rho(k) Y, H) d L\left(X_{i}\right) f(k) .
$$

The lemma now follows from (*).

4. The basic theorem. We now specialize the construction of the last section to the generalized Lorentz groups. Let $\boldsymbol{n}$ be an integer, equal to or greater than 2 . Let $G=G(n)$ or $\hat{G}(n)$, where $G(n)$ is the identity component of $\operatorname{SO}(1, n)$, and $\hat{G}(n)$ is the group $\operatorname{Spin}(1, n)$. The latter group is a two-fold covering group of $G(n)$, and is simply connected if $n \geq 3$. The group $G(n)$ is the identity component of the orthogonal group of a quadratic form $Q$, defined on a real $n+1$-dimensional vector space, where $Q$ is nondegenerate, and has signature equal to $-n$. In a canonical basis, this form has the matrix $J=\operatorname{diag}[1,-1, \ldots,-1]$. In such a basis, $G(n)$ may be identified with a set of matrices $A$ which satisfy the relation $A^{T} J A=J$. We may take $K=K(n)$ to be the subgroup which fixes the vector $(1,0, \ldots, 0)$. This subgroup is isomorphic to $\mathrm{SO}(n)$. The corresponding Cartan decomposition may be written $\mathbf{G}(n)=\mathbf{K}(n) \oplus \mathbf{P}(n)$, where $\mathbf{K}(n)$ is the set of skew symmetric matrices given by the expression

$$
\mathbf{K}(n)=\operatorname{span}_{\mathbf{R}}\left\{E_{i j}-E_{j i} \mid 1 \leq i \leq j \leq n\right\},
$$

and the Cartan subspace $\mathbf{P}=\mathbf{P}(n)$ is given by the set of symmetric matrices

$$
\mathbf{P}(n)=\operatorname{span}_{\mathbf{R}}\left\{E_{0 i}+E_{i 0} \mid i=1, \ldots, n\right\},
$$

where $E_{i j}$, for $0 \leq i, j \leq n$, are the matrix units in the canonical basis. We write $\mathbf{N}(n), \mathbf{S}(n), \mathbf{A}(n)$, respectively, for the subalgebras which in this case correspond to the subalgebras $\mathbf{N}, \mathbf{S}$, and $\mathbf{A}$ of the last section. Similarly, we denote by $N(n)$, $S(n), A(n)$, and $K(n)$ the subgroups of $G(n)$ which correspond, respectively, to the subgroups $N, S, A$, and $K$ of the last section. The corresponding subgroups of the 
spinor group $\hat{G}(n)$ are denoted by $\hat{N}(n), \hat{S}(n), \hat{A}(n)$, and $\hat{K}(n)$, respectively.

For $G(n)$ we have $r_{1}=r=n-1$. Hence, for these groups, the last term on the right-hand side of the equation in Lemma 1 is absent.

In order to derive a more useful form of this lemma, as well as to be able to state our main results, we make some known comments concerning the weights of irreducible representations of $\mathbf{K}(n)$.

Let $p$ be the rank of $\mathbf{K}(n)$. Then $n=2 p$, or $n=2 p+1$. Let $\mathbf{H}(n)$ be a Cartan subalgebra of $\mathbf{K}(n)$. It is convenient to extend the inner product (, ), defined on $\mathbf{G}$, to an Hermitian inner product on $\mathbf{G}_{\mathbf{C}}$ by means of the definition $\left(x+i x^{\prime}, y\right.$ $\left.+i y^{\prime}\right)=(x, y)+\left(x^{\prime}, y^{\prime}\right)$, for $x, x^{\prime}, y^{\prime}, y \in \mathbf{G}(n)$. We identify $\mathbf{H}(n)_{\mathbf{c}}$ with its dual by means of the form $($,$) . Let \left(i \varepsilon_{1}, \ldots, i \varepsilon_{p}\right)$ be an ordered orthonormal basis of $\mathbf{H}(n)_{\mathbf{c}}$. The weights of any finite-dimensional representation of $\mathbf{K}(n)$ are pure imaginary forms on $\mathbf{H}(n)$. In particular, let $\Sigma$ be the set of simple positive roots, corresponding to the lexicographical ordering on $i \mathbf{H}(n)$ determined by the above ordered basis. Then

$$
\Sigma=\left\{\varepsilon_{1}-\varepsilon_{2}, \ldots, \varepsilon_{2 p-1}-\varepsilon_{2 p}, \varepsilon_{2 p}\right\},
$$

in case $n=2 p+1$, and in case $n=2 p$,

$$
\Sigma=\left\{\varepsilon_{1}-\varepsilon_{2}, \ldots, \varepsilon_{2 p-1}-\varepsilon_{2 p}, \varepsilon_{2 p-1}+\varepsilon_{2 p}\right\} .
$$

Let [ $\omega]$ be a class in $\Omega(R(n))$, the set of equivalence classes of irreducible unitary representations of $\hat{K}(n)$. Let $\Lambda_{\omega}$ be the highest weight corresponding to this class. We may write $\Lambda_{\omega}=\sum_{i=1}^{p} \Lambda_{\omega i} \varepsilon_{i}$. In terms of this basis, the standard dominance conditions:

$$
2\left(\Lambda_{\omega}, \alpha\right) /(\alpha, \alpha) \geq 0,
$$

where $\alpha \in \Sigma$, and the numbers on the left are integers, become

$$
\Lambda_{\omega 1} \geq \Lambda_{\omega 2} \geq \ldots \geq \Lambda_{\omega p-1} \geq\left|\Lambda_{\omega p}\right|,
$$

in case $n=2 p$, and in case $n=2 p+1$,

$$
\Lambda_{\omega 1} \geq \Lambda_{\omega 2} \geq \ldots \geq \Lambda_{\omega p} \geq 0 .
$$

The components of the highest weights must be integers or half odd integers. The latter representations correspond to the spinor representations which do not correspond to "single-valued" representations of $K(n)$. The weights of the irreducible representation $\left[\mathbf{P}(n)_{\mathbf{C}}, d \rho\right]$ are given by the expression $\left\{ \pm \varepsilon_{1}, \ldots, \pm \varepsilon_{p}\right\}$, in case $n=2 p$, and by the expression $\left\{ \pm \varepsilon_{1}, \ldots, \pm \varepsilon_{p}, 0\right\}$ in case $n=2 p+1$. Let $\delta$ be the form defined as $2 \delta=$ sum of positive roots of $\mathbf{K}(n)$. Then for $n=2 p$ we have $2 \delta=2\left[(p-1) \varepsilon_{1}+(p-2) \varepsilon_{2}+\ldots+\varepsilon_{p-1}\right]$, and for $n=2 p+1$ we have $2 \delta=(2 p-1) \varepsilon_{1}+(2 p-3) \varepsilon_{2}+\ldots+3 \varepsilon_{p-1}+\varepsilon_{p}$. 
Finally, for each $Y \in \mathbf{P}(n)$ we write $\phi_{Y}$ for the function $k \rightarrow(\rho(k) y, H)$. These functions are defined on the right coset spaces $\hat{M}(n) \backslash \hat{K}(n) \simeq M(n) \backslash K(n)$, that is, the $n-1$-sphere. They are in fact spherical harmonics of degree one.

Theorem 1. Let $f \in L_{\Lambda}^{2}(\hat{G}(n), \underline{H})$, where $\underline{H}$ is a finite-dimensional inner product space, and let $Y \in \mathbf{P}(n)_{\mathbf{C}}$. Then, unless $\Lambda_{\omega^{\prime}}-\Lambda_{\omega}$ is a weight in $\left[\mathbf{P}_{\mathbf{C}}, d \rho\right]$, we have $E_{\omega^{\prime}} d R(Y) E_{\omega} f=0$. Now assume that $\Lambda_{\omega^{\prime}}-\Lambda_{\omega}=\sigma$ is a weight in $\left[\mathbf{P}_{\mathbf{C}}, d \rho\right]$. Then we have

$$
E_{\omega^{\prime}} d R(Y) E_{\omega} f=\left(\lambda-(n-1) / 2+(\sigma, \sigma) / 2+\left(\sigma, \Lambda_{\omega}+\delta\right)\right) E_{\omega^{\prime}} \phi_{Y} E_{\omega} f
$$

The theorem follows easily from Lemma 1 and some known observations concerning irreducible $\mathbf{K}(n)$-modules.

Lemma 2. Let $\left[H_{\omega}, \omega\right]$ be a representation belonging to the class $[\omega]$ in $\Omega(\hat{K}(n))$. Then the eigenvalue of $\Omega_{K}$ in this representation is given by

$$
d \omega\left(\Omega_{K}\right)=\left[\left(\Lambda_{\omega}+\delta, \Lambda_{\omega}+\delta\right)-(\delta, \delta)\right] 1,
$$

where 1 is the identity function on $H_{\omega}$.

Proof. This formula is true for any compact or complex semisimple Lie algebra. See [13, p. 247]. For $n=2$, the result is true with $\delta=0$.

Lemma 3. Consider the reduction of the tensor product representation $\left[H_{\omega}\right.$ $\left.\otimes \mathbf{P}(n)_{\mathbf{C}}, d \rho \otimes \omega\right]$ into irreducible representations. $A$ class $\left[\omega^{\prime}\right]$ occurs in this reduction if and only if the highest weight $\Lambda_{\omega^{\prime}}$ satisfies the condition that $\Lambda_{\omega^{\prime}}-\Lambda_{\omega}$ $=\sigma$ is a weight in $\left[\mathbf{P}(n)_{\mathbf{C}}, d \rho\right]$.

Proof. This lemma follows immediately from the general theory of reduction of tensor product representations. See [3] or [13, p. 262].

Proof of the theorem. For each class $[\omega] \in \Omega(\hat{K}(n))$, the map $Y \otimes E_{\omega} f$ $\rightarrow \phi_{Y} E_{\omega} f$ is a $\mathbf{K}(n)$-module homomorphism of the $\mathbf{K}(n)$ module $\mathbf{P}(n)_{\mathbf{C}}$ $\otimes E_{\omega} L_{\Lambda}^{2}(G(n))$ into $L_{\Lambda}^{2}(G(n))$. In the representation $\left[\mathbf{P}(n)_{\mathbf{C}}, d \rho\right]$, the eigenvalue of $d \rho\left(\Omega_{K}\right)$ is $\mu=(n-1)$. Hence, the theorem follows.

5. Some remarks on the $\hat{K}(n)$-module structure of the induced representations. Let $[\underline{H}, \Lambda \mu]$ be a finite-dimensional representation of the parabolic subgroup $\hat{S}(n) \hat{M}(n)$. We point out that the $\hat{K}(n)$-module structure of the space $d C_{\Lambda \mu}(\hat{K}(n))$ can be completely determined from the Frobenius reciprocity theorem for compact groups, and the branching theorem. (See [2, p. 248].)

We note that the subgroup $\hat{M}(n)$ is isomorphic to $\operatorname{Spin}(n-1)$, if $n \geq 3$. The group $\operatorname{Spin}(2)$ is the circle group. For $n=2, \hat{G}(n) \simeq \operatorname{SL}(2, \mathbf{R})$. In this case, $\hat{K}(n)$ is the circle group, and $\hat{M}(n)$ is the two element group comprising the center of $\hat{G}(n)$. In case $n=2 p+1, \operatorname{rank}(\mathbf{K}(n))=\operatorname{rank}(\mathbf{M}(n))=p$. In case $n=2 p$, the rank of $\mathbf{M}(n)$ is $p-1$. In the latter case, choose the basis $\left\{\varepsilon_{1}, \ldots, \varepsilon_{p}\right\}$ in such a manner 
that the first $p-1$ elements lie in the subalgebra $\hat{M}(n)$. We call a representation $[\omega] \in \Omega(\hat{K}(n))$, or a representation class $[\mu] \in \Omega(\hat{M}(n))$, a spinor (ordinary) representation, in case the components of the highest weights in the above bases are half odd integers (integers). In case $n=2$, the nontrivial (trivial) character of $\hat{M}(n)$ is called the spinor (ordinary) representation. With these definitions, the spinor representations are precisely the ones whose kernels do not contain the kernel of the covering map $\operatorname{Spin}(n) \rightarrow \mathrm{SO}(n)$.

Lemma 4. Consider the restriction of the representations $\left[L_{\Lambda \mu}^{2}(\hat{G}(n), \underline{H}), R\right]$ and $\left[C_{\Lambda \mu}(\hat{G}(n), \underline{H}), R\right]$ to the subgroup $K(n)$. Then the following statements are true. (1) The class $[\omega] \in \Omega(\hat{K}(n))$ occurs in these restrictions with the same multiplicity as $[\mu]$ occurs in the restriction of $[\omega]$ to $\hat{M}(n)$. (2) If $[\mu]$ is a spinor (ordinary) representation of $\hat{M}(n)$, then every class $[\omega]$ that occurs in the above restricted representation is a spinor (ordinary) representation, and in case $n \geq 3$, the components of the highest weights $\Lambda_{\omega}$, and $\Lambda_{\mu}$, satisfy the following inequalities:

(a) for $n=2 p$,

$$
\Lambda_{\omega 1} \geq \Lambda_{\mu 1} \geq \Lambda_{\omega 2} \geq \Lambda_{\mu 2} \geq \Lambda_{\omega 3} \geq \ldots \geq \Lambda_{\mu p-1} \geq\left|\Lambda_{\omega p}\right|
$$

(b) for $n=2 p+1$,

$$
\Lambda_{\omega 1} \geq \Lambda_{\mu 1} \geq \Lambda_{\omega 2} \geq \Lambda_{\mu 2} \geq \ldots \geq \Lambda_{\mu p-1} \geq \Lambda_{\omega p} \geq\left|\Lambda_{\mu p}\right|
$$

Moreover, if $\Lambda_{\omega}$ satisfies condition (7a) or (7b) above, then [ $\left.\omega\right]$ occurs with multiplicity 1 in the induced representation.

Proof. Statement (2) follows immediately from statement (1) and the branching theorem referred to above. By the discussion in $\$ 3$ the restriction-to- $R(n)$ map is a $\hat{K}(n)$-module isomorphism. The image of this map is the induced representation of $\hat{R}(n)$ induced by the representation $[\underline{H}, \mu]$ of the subgroup $\hat{M}(n)$. The lemma follows then by the Frobenius reciprocity theorem for compact groups. (See Weil [19, p. 82].) Q.E.D.

6. The first irreducibility theorem. Let $[\underline{H}, \mu]$ be an irreducible representation of the subgroup $\hat{M}(n)$. We consider now the induced representation $\left[L_{\Lambda \mu}^{2}(\hat{G}(n)), R\right]$ induced by the representation $\Lambda P^{-1} \mu$ of the parabolic subgroup $\hat{S}(n) \hat{M}(n)$. As before $L_{\Lambda \mu}^{2}(\hat{G}(n), \underline{H})$ may be viewed as a closed invariant subspace of $L_{\Lambda}^{2}(\hat{G}(n), \underline{H})$.

As a first application of the main theorem, we derive sufficient conditions for the irreducibility of this module. We recall that topological irreducibility of the Banach space representations, belonging to the infinitesimal equivalence class of the module $\left[L_{\Lambda \mu}^{2}(\hat{G}(n), \underline{H}), R\right]$, is equivalent to algebraic irreducibility of the $\mathbf{K}(n)$ module $\left[d C_{\Lambda \mu}(\hat{G}(n), \underline{H}), d R\right]$.

Theorem 2. Suppose that $[\underline{H}, \mu]$ is an ordinary representation of $\hat{M}(n)$, and suppose that the number $\lambda=d \Lambda(H)$ is not equal to an integer. Then, $\left[d C_{\Lambda \mu}(\hat{G}(n), \underline{H}), d R\right]$ is irreducible. 
Suppose that $[\underline{H}, \mu]$ is a spinor representation of $\hat{M}(n)$. Then the module $\left[d C_{\Lambda \mu}(\hat{G}(n), \underline{H}), d R\right]$ is irreducible provided $\lambda$ is not equal to a half odd integer.

Finally, $d C_{\Lambda \mu}(\hat{G}(n), \underline{H})$ is an irreducible $U(\mathbf{G}(n))$-module if for all classes $[\omega]$ occurring in the restriction to $\hat{K}(n)$, the following conditions hold: $\lambda+\Lambda_{\omega i}+1-i$ $\neq 0, \lambda-\Lambda_{\omega i}-n+1 \neq 0,1 \leq i \leq p$.

Proof. Under the hypothesis of the theorem we will show that every nonzero element of the space $d C_{\Lambda \mu}(\hat{G}(n), \underline{H})$ is cyclic. Let $f$ be a nonzero element. Let $\underline{V}$ be the $U(\mathbf{G}(n))$-module generated by $f$; that is, $\underline{V}=d R(U(\mathbf{G}(n))) f$.

It is known that the universal enveloping algebra has the following decomposition (see [9a, part I]):

$$
U(\mathbf{G}(n))=U(\mathbf{P}(n)) U(\mathbf{K}(n))
$$

where $U(\mathbf{P}(n))$ is the image in $U(\mathbf{G}(n))$ of the symmetric algebra $S(\mathbf{P}(n))$, under the symmetrization map. Let $F$ be the subspace of $d C_{\Lambda \mu}(\hat{G}(n), \underline{H})$ defined by $F=d R(U(\mathbf{K}(n))) f$. This subspace is obviously finite dimensional and a $\mathbf{K}(n)$ module. Moreover, $V=d R(U(\mathbf{P}(n))) F$.

Let $\delta$ be the pointwise $\mathbf{C}$-algebra generated by 1 , and the functions $g \rightarrow \phi_{Y}(g)$ $=(\rho(\kappa(g)) Y, H)$, where $Y \in \mathbf{P}(n)_{\mathbf{c}}$. We recall that $\kappa$ is the analytic projection of $\hat{G}(n)$ onto the subgroup $\hat{K}(n)$ defined by the Iwasawa decomposition. This algebra consists of the spherical harmonics defined on the coset space $\hat{M}(n) \backslash \hat{K}(n)$. We show that it consists of all the $\hat{K}(n)$-finite functions defined on this coset space. In fact, $\delta$ is closed under complex conjugation, since it is generated by real functions. Moreover, this algebra separates points as the following observations show: Assume that for all $Y \in \mathbf{P}(n)_{\mathbf{C}}$ we have $\left(\rho\left(k_{1}\right) Y, H\right)$ $=\left(\rho\left(k_{2}\right) Y, H\right)$. Then since $\rho$ is unitary, we have $\left(Y, \rho\left(k_{1}^{-1}\right) H\right)=\left(Y, \rho\left(k_{2}^{-1}\right) H\right)$, for all $Y \in \mathbf{P}(n)$. From the nondegeneracy of the inner product ( , ), it follows that $\rho\left(k_{1}^{-1}\right) H=\rho\left(k_{2}^{-1}\right) H$, or that $k_{1} k_{2}^{-1}$ fixes the element $H$, and hence is in $\hat{M}(n)$. In other words, $k_{1}$ and $k_{2}$ lie in the same right coset. Hence the assertion follows from the Stone-Weierstrass theorem.

Now, by the argument used in Lemma 3 of [18] it follows that the space $d C_{\Lambda \mu}(\hat{G}(n), \underline{H})$ is generated as an $\delta$-module by any finite-dimensional $\hat{K}(n)$ submodule of $d C_{\Lambda \mu}(\hat{G}(n), \underline{H})$. In particular we have

$$
\delta \ni=d C_{\Lambda \mu}(\hat{G}(n), \underline{H}) .
$$

Hence, the proof of the theorem will be complete once it is shown that $V$ is an $\delta$-module, for in that case we will have

$$
d C_{\Lambda \mu}(\hat{G}(n), \underline{H})=\delta \ni \subseteq \nu=d R(U(G(n))) f .
$$

$V$ is a direct sum of irreducible $\hat{K}(n)$-modules. Hence, for all $[\omega] \in \Omega(\hat{K}(n))$ we have $E_{\omega} \nu \subset \mathcal{V}$. Assume $[\omega]$ occurs in the $\hat{K}(n)$-module reduction of $v$. By 
Theorem 1, we have, for all $Y \in \mathbf{P}_{\mathbf{C}}(n)$,

$$
E_{\omega^{\prime}} d R(Y) E_{\omega}=q_{\omega^{\prime} \omega} E_{\omega^{\prime}} \phi_{Y} E_{\omega},
$$

where $q_{\omega^{\prime} \omega}=0$ unless $\Lambda_{\omega^{\prime}}-\Lambda_{\omega}=\sigma$, a weight in $\left[\mathbf{P}_{\mathbf{C}}(n), d \rho\right]$. We note that $E_{\omega^{\prime}} \phi_{Y} E_{\omega}=0$ as well, unless $\left[\omega^{\prime}\right]$ belongs to the tensor product $\rho \otimes \omega$. Hence $E_{\omega^{\prime}} \phi_{Y} E_{\omega}=0$ unless $\Lambda_{\omega^{\prime}}-\Lambda_{\omega}=\sigma$, a weight in $\left[\mathbf{P}_{\mathbf{C}}(n), d \rho\right]$, by Lemma 3 .

On the other hand, if $\left[\omega^{\prime}\right]$ does occur in the tensor product $\rho \otimes \omega$, we assert that $q_{\omega^{\prime} \omega} \neq 0$. The theorem will follow from this assertion, since then $E_{\omega^{\prime}} \phi_{Y} E_{\omega} \nu$

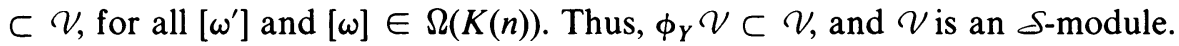

By Theorem 1, we have

$$
\begin{aligned}
q_{\omega^{\prime} \omega} & =\lambda+\Lambda_{\omega i}+1-i, & & \text { in case } \sigma=\varepsilon_{i}, \\
& =\lambda-\Lambda_{\omega i}-n+i+1, & & \text { in case } \sigma=-\varepsilon_{i}, \\
& =\lambda-p, & & \text { in case } \sigma=0,
\end{aligned}
$$

the last condition applies only to the case $n=2 p+1$.

Now by Lemma 4 if $[\mu]$ is a spinor representation, then $\Lambda_{\omega i}$ is a half odd integer, for all $1 \leq i \leq p$, and in case $[\mu]$ is an ordinary representation, $\Lambda_{\omega i}$ is an integer. In all cases the hypothesis of the theorem insures that $q_{\omega^{\prime} \omega} \neq 0$. Thus the assertion follows. Q.E.D.

7. A lemma for the nonirreducible cases. In order to examine the situation in which the hypothesis of Theorem 2 fails to be satisfied, it is convenient to introduce a class of commutative operator algebras, which plays the same role that the algebra $\delta$ plays in the proof of Theorem 2 .

We shall introduce the following terminology. Let $[\mu]$ be an irreducible representation of the subgroup $\hat{M}(n)$, and let $\Lambda_{\mu}$ be its highest weight. Let us say that an irreducible representation of $\hat{K}(n),[\omega]$, is $\mu$-admissible if this representation occurs in the induced representation of $\hat{K}(n)$ induced by the representation $[\mu]$. It follows immediately from Lemma 4 that this condition is equivalent to the condition that the components of the highest weight $\Lambda_{\omega}$ satisfy the inequalities (7).

Let $\Omega_{\mu}(\hat{K}(n))$ be the subset of $\Omega(\hat{K}(n))$ consisting of $\mu$-admissible representation classes. Suppose $D$ is a subset of $\Omega_{\mu}(\hat{K}(n))$, and let $[\underline{H}, \Lambda \mu]$ be an irreducible $\hat{S}(n) \hat{M}(n)$-module corresponding to the character $\Lambda$ of $\hat{S}(n)$ and the representation $\mu$ of $\hat{M}(n)$. Then we define an Hermitian projection $D$ on the Hilbert space $L_{\Lambda \mu}^{2}(G, \underline{H})$ by means of the formula

$$
D=\sum\left\{E_{\omega}:[\omega] \in D\right\}
$$

The above formula defines a Hilbert space projection, since the projections $E_{\omega}$, $[\omega] \in \Omega_{\mu}(\hat{K}(n))$ are mutually orthogonal, by the Schur orthogonality relations. If $f \in d C_{\Lambda \mu}(\hat{G}(n), \underline{H})$, then only a finite number of terms in the sum 


$$
D f=\sum\left\{E_{\omega} f:[\omega] \in D\right\}
$$

are different from zero. Hence $D f$ is also a $K$-finite vector. Since $d C_{\Lambda \mu}(\hat{G}(n), \underline{H})$ is dense in $L_{\Lambda \mu}^{2}(\hat{G}(n), \underline{H})$, it now follows easily that $D d C_{\Lambda \mu}(\hat{G}(n), \underline{H})$ is dense in $D L_{\Lambda \mu}^{2}(\hat{G}(n), \underline{H})$.

Now let $\delta_{D}$ be the C-algebra of operators on $L_{\Lambda \mu}^{2}(\hat{G}(n), \underline{H})$ generated by the operators $D \phi_{Y} D$, for $Y \subset \mathbf{P}_{\mathbf{C}}(n)$. The functions $\phi_{Y}$ are of course regarded as multiplication operators on $L_{\Lambda \mu}^{2}(\hat{G}(n), \underline{H})$. When $D=1$, or equivalently, when $D=\Omega_{\mu}(R(n))$, the algebra $\delta_{1}$ is of course equivalent to the algebra $\delta$, introduced in the proof of Theorem 2, when the latter is regarded as an algebra of multiplication operators on $L_{\Lambda \mu}^{2}(\hat{G}(n), \underline{H})$.

Lemma 5. Let $D$ be a set of $\mu$-admissible representation classes, and let $D$ be the Hermitian projection that corresponds to this set, as defined above. Let $\exists$ be a $\hat{K}(n)-$ invariant finite-dimensional subspace of $d C_{\Lambda \mu}(\hat{G}(n), \underline{H})$, and assume that $D \Im=\Im$. Assume also that $D$ has the following property: If $[\omega]$ and $\left[\omega^{\prime}\right] \in D$, then $D$ contains all the $\mu$-admissible classes which lie between $[\omega]$ and $\left[\omega^{\prime}\right]$ in the lexicographical ordering of highest weights. Then we have the following equality of subspaces:

$$
\delta_{D} \ni=D \delta_{1} \Im=D d C_{\Lambda \mu}(\hat{G}(n), \underline{H}) \text {. }
$$

Proof. The equality $D \delta_{1} \exists=D d C_{\Lambda \mu}(\hat{G}(n), \underline{H})$ follows immediately from the equality $\delta \exists=d C_{\Lambda \mu}(\hat{G}(n), \underline{H})$, which was pointed out in the proof of Theorem 2 . It remains to prove the first inequality. It clearly suffices to assume that $\exists$ is $\hat{K}(n)$-irreducible and belongs to the $\mu$-admissible class [ $\omega]$. Let

$$
\mathbf{C}=\delta_{D}^{0} \subset \delta_{D}^{1} \subset \ldots \subset \delta_{D}^{t} \ldots, \quad \mathbf{C}=\delta^{0} \subset \delta^{1} \subset \ldots \subset \delta^{t} \ldots
$$

denote the standard filtrations that the algebras $\delta_{D}$ and $\delta$ acquire as algebras of polynomials. We shall prove that for all degrees $t, t \geq 0$,

$$
\delta_{D}^{t} \ni=D \delta^{t} \Im
$$

Since, for all $t, t \geq 0$, the linear space $\delta^{t} \ni$ is equivalent to a direct sum of its $\hat{K}(n)$-irreducible subspaces, it follows immediately that $\delta_{D}^{t} \Im \subseteq D \delta^{t} \Im$ for all $t$, $t \geq 0$. Hence it is sufficient to show that $\delta_{D}^{t} \Im \supseteq D \delta^{t} \Im$. The proof proceeds by induction on the degree $t$. The last statement is obvious for $t=0$. Let us assume that this statement is true for $t=u \geq 0$.

We note that the linear space $D \delta^{u+1} \Im$ is generated by the subspace $D \delta^{u} \Im$ and the set of functions $D \phi_{Y} \phi^{\prime}$, with $\phi^{\prime} \in \mathcal{\delta}^{u} \Im$ and $Y \in \mathbf{P}_{\mathbf{C}}(n)$. Hence, it suffices to show that $D \phi_{Y} \phi^{\prime} \in \delta_{D}^{u+1} \Im$, for $\phi^{\prime} \in \mathcal{S}^{u} \Im$, and $Y \in \mathbf{P}_{\mathbf{C}}(n)$.

We may write

$$
D \phi_{Y} \phi^{\prime}=D \phi_{Y} D \phi^{\prime}+D \phi_{Y}(1-D) \phi^{\prime}
$$


By induction hypothesis, $D \phi^{\prime} \in \delta_{D}^{u} \ni$. Hence the first term on the right-hand side of the last equation lies in $\delta_{D}^{u+1} \Im$. If the second term is equal to zero we are done. Assume that $D \phi_{Y}(1-D) \phi^{\prime} \neq 0$. This expression is a sum of terms of the form $E_{\omega^{\prime}} \phi_{Y} E_{\omega^{\prime \prime}} \phi^{\prime}$, with $\left[\omega^{\prime}\right] \in D$ and $\left[\omega^{\prime \prime}\right] \notin D$, and $\Lambda_{\omega^{\prime}}=\Lambda_{\omega^{\prime \prime}}+\sigma$, with $\sigma= \pm \varepsilon_{j}$ for some $j, 1 \leq j \leq p$. The last condition follows from Lemma 3. We assert that $E_{\omega^{\prime}} \phi_{Y} E_{\omega^{\prime \prime}} \phi^{\prime}$ lies in the subspace $\mathcal{S}^{u} \Im$. Whence the lemma follows by the induction hypothesis, once this assertion is proved.

It follows from the Frobenius reciprocity theorem and the branching theorem that each $\mu$-admissible representation class occurs with multiplicity one in the $\hat{K}(n)$-module $\left[d C_{\Lambda \mu}(\hat{G}(n), \underline{H}), R\right]$. Now it follows from Lemma 3, and also from the branching theorem, that the $\mu$-admissible representations have highest weight $\Omega$ which lie on certain lattice points in a $p$-dimensional lattice. These lattice points correspond to vectors $\Omega$ such that the components of $\Omega-\Lambda_{\omega}$, in the basis $\left\{\varepsilon_{j}\right\}$, are integers. Lemma 3 tells us more: Let us look at the sequence of subspaces $\exists, \delta^{1} \exists, \delta^{2} \exists, \ldots$ As one moves along this sequence, the lattice points which correspond to $\mu$-admissible representations "fill in" without gaps in any direction. In other words, the lattice points whose $j$ th coordinates are $\Lambda_{\omega j} \pm s$ must fill in before the lattice points whose $j$ th coordinates are $\Lambda_{\omega, j} \pm(s+1), s \geq 0$. In particular, we see that the lattice point corresponding to $\Lambda_{\omega^{\prime}}$ must have been filled in before the lattice point corresponding to $\Lambda_{\omega^{\prime \prime}}$. It follows that

$$
E_{\omega^{\prime}} \phi_{Y} E_{\omega^{\prime \prime}} \phi^{\prime \prime} \in \mathcal{\delta}^{u} \Im \text {. Q.E.D. }
$$

Remark. In the course of the above argument we have shown that for some $Y \neq 0, E_{\omega^{\prime}} \phi_{Y} E_{\omega^{\prime \prime}} \neq 0$, whenever $\Lambda_{\omega^{\prime}}-\Lambda_{\omega^{\prime \prime}}$ is a nonzero weight in $\left[\mathbf{P}_{\mathbf{C}}(n), d \rho\right]$ and $\omega^{\prime}$ and $\omega^{\prime \prime}$ are $\mu$-admissible.

8. The nonirreducible cases. We shall now investigate the situation in which the hypothesis of Theorem 2 fails to be satisfied. We shall perform a case by case analysis of this situation.

Theorem 3. Fix a real number $\lambda=d \Lambda(H)$. Suppose there exists an index $i$, $1 \leq i \leq p$, and a $\mu$-admissible class $[\omega] \in \Omega_{\mu}(\hat{K}(n))$ such that $\lambda+\Lambda_{\omega i}-i+1$ $=0$. Let $s$ be the integer or half odd integer defined by the equation $\lambda+s-i+1$ $=0$. Assume also that either $i \neq p$ or that $n=2 p+1$. Then the index $i$ is unique. Let $D_{\lambda}^{-}$be the subset of $\Omega_{\mu}(\hat{K}(n))$ defined by

$$
D_{\lambda}^{-}=\left\{\left[\omega^{\prime}\right] \in \Omega_{\mu}(\hat{K}(n)): \Lambda_{\omega^{\prime} i} \leq s\right\}
$$

Then the following statements are true.

(1) The projection $D_{\lambda}^{-}=\sum\left\{E_{\omega}:[\omega] \in D_{\lambda}^{-}\right\}$is an invariant projection; that is, $D_{\lambda}^{-} d C_{\Lambda \mu}(\hat{G}(n), \underline{H})$ is a $d R(U(\mathbf{G}(n)))$-module.

(2) The $d R(U(\mathbf{G}(n)))$-module in statement (1) is irreducible.

(3) The projection defined in (1) has no supplementary invariant subspaces. In 
particular, the submodule defined in (2) is a unique irreducible submodule of $\left[d C_{\Lambda \mu}(\hat{G}(n), \underline{H}), d R\right]$.

Proof. First, we note that the index $i$ defined in the hypothesis is unique. Suppose that for some $\left[\omega^{\prime}\right]$ and $j \neq i, \lambda+\Lambda_{\omega^{\prime} j}-j+1=0$. Then $\Lambda_{\omega^{\prime} j}=s+j$ $-i$. If $j>i$, then by the inequalities (7), we have $s \geq \Lambda_{\mu i} \geq \Lambda_{\mu j-1} \geq s+j-i$, a contradiction. Similarly, if $j<i$, we have $s+j-i \geq \Lambda_{\mu j} \geq \Lambda_{\mu-1} \geq s$, again a contradiction.

Now, we will show that under the conditions of the theorem, $D_{\lambda}^{-} d C_{\Lambda \mu}(\hat{G}(n), \underline{H})$ is an invariant subspace. Since the projection $D_{\lambda}^{-}$is invariant under the restriction of the representation to $\hat{K}(n)$, it is sufficient to show that $D_{\lambda}^{-} d R(Y) D_{\lambda}^{-}$ $=d R(Y) D_{\lambda}^{-}$, for all $Y \in \mathbf{P}_{\mathbf{C}}(n)$. This condition is equivalent to the condition that $\left(1-D_{\lambda}^{-}\right) d R(Y) D_{\lambda}^{-}=0$, for all $Y \in \mathbf{P}_{\mathbf{C}}(n)$. If $1=D_{\lambda}^{-}$, the latter condition follows trivially. To prove the last condition when $1 \neq D_{\lambda}^{-}$, we will show that for any $\mu$ admissible classes $\left[\omega^{\prime}\right]$ and $\left[\omega^{\prime \prime}\right]$ such that $\left[\omega^{\prime}\right] \in D_{\lambda}^{-}$and $\left[\omega^{\prime \prime}\right] \notin D_{\lambda}^{-}$we have $E_{\omega^{\prime \prime}} d R(Y) E_{\omega^{\prime}}=0$, for all $Y \in \mathbf{P}_{\mathbf{C}}(n)$.

Assume that $\left[\omega^{\prime}\right] \in D_{\lambda}^{-}$and $\left[\omega^{\prime \prime}\right] \notin \mathcal{D}_{\lambda}^{-}$. If $\Lambda_{\omega^{\prime \prime}}-\Lambda_{\omega^{\prime}}$ is not a weight in $\left[\mathbf{P}_{\mathbf{C}}(n), d \rho\right]$, then it follows from Lemma 3 that $E_{\omega^{\prime \prime}} d R(Y) E_{\omega^{\prime}}=0$. Suppose that $\Lambda_{\omega^{\prime \prime}}-\Lambda_{\omega^{\prime}}$ is a weight in $\left[\mathbf{P}_{\mathbf{C}}(n), d \rho\right]$. Then by Lemma 3 , we must have, for some $j, 1 \leq j \leq p, \Lambda_{\omega^{\prime \prime}}=\Lambda_{\omega^{\prime}} \pm \varepsilon_{j}$. On the other hand, by the definition of the set $D_{\lambda}^{-}$, we must have $\Lambda_{\omega^{\prime \prime} i}>s$ and $\Lambda_{\omega^{\prime} i} \leq s$. Hence, by Theorem 1 we have, for all $Y \in \mathbf{P}_{\mathbf{C}}(n)$

$$
E_{\omega^{\prime \prime}} d R(Y) E_{\omega^{\prime}}=[\lambda+s+1-i] E_{\omega^{\prime \prime}} \phi_{Y} E_{\omega^{\prime}}=0 .
$$

Hence $\left(1-D_{\lambda}^{-}\right) d R(Y) D_{\lambda}^{-}=0$ for all $Y \in \mathbf{P}_{\mathbf{C}}(n)$.

In order to prove the irreducibility of the module $D_{\lambda}^{-} d C_{\Lambda \mu}(\hat{G}(n), \underline{H})$, we will show that an arbitrary nonzero element $f$ in this space is cyclic. Let $f$ be such a nonzero element. Let $\exists$ be the $U(\mathbf{K}(n))$-module generated by $f$. Since $f$ is $\hat{K}(n)$ finite, $\Im$ is finite dimensional. Hence, by Lemma 5 , irreducibility is proved once we have shown that

$$
\delta_{D_{\lambda}^{-}} \subseteq \subseteq d R\left(U\left(\mathbf{P}_{\mathbf{C}}(n)\right)\right) \exists .
$$

The last inclusion will be proved once we have shown that the space on the right of the inclusion is an $\delta_{D_{\lambda}^{-}}$-module.

Let $g^{\prime} \in d R(U(\mathbf{P}(n))) \rightarrow, g^{\prime} \neq 0$. Then for $Y \in \mathbf{P}_{\mathbf{C}}(n), E_{\omega^{\prime \prime}} d R(Y) E_{\omega^{\prime}} g^{\prime}=0$ unless $\left[\omega^{\prime \prime}\right],\left[\omega^{\prime}\right] \in \mathcal{D}_{\lambda}^{-}$, by the first part of the theorem. Assume $\left[\omega^{\prime \prime}\right],\left[\omega^{\prime}\right] \in D_{\lambda}^{-}$. Then, as in the proof of Theorem 2 we have

$$
E_{\omega^{\prime \prime}} d R(Y) E_{\omega^{\prime}} g^{\prime}=q_{\omega^{\prime \prime} \omega^{\prime}} E_{\omega^{\prime \prime}} \phi_{Y} E_{\omega^{\prime}} g^{\prime},
$$

where $q_{\omega^{\prime \prime} \omega^{\prime}}=0$ unless $\Lambda_{\omega^{\prime \prime}}-\Lambda_{\omega^{\prime}}=\sigma$, a weight in $\left[\mathbf{P}_{\mathbf{C}}(n), d \rho\right]$. In that case we have 


$$
\begin{aligned}
q_{\omega^{\prime \prime} \omega^{\prime}} & =\lambda-\Lambda_{\omega^{\prime} j}-n+j+1 & & \text { in case } \sigma=-\varepsilon_{j}, \\
& =\lambda+\Lambda_{\omega^{\prime} j}+1-j & & \text { in case } \sigma=\varepsilon_{j}, \\
& =\lambda-p & & \text { in case } \sigma=0 .
\end{aligned}
$$

The last case is applicable only when $n=2 p+1$.

In the first case we find that $\Lambda_{\omega^{\prime \prime} j}=\Lambda_{\omega^{\prime} j}-1$; so that

$$
\begin{aligned}
q_{\omega^{\prime \prime} \omega^{\prime}} & =\lambda-\Lambda_{\omega^{\prime} j}-n+j+1<\lambda-\Lambda_{\omega^{\prime \prime} j}-n+j+1 \\
& =-\Lambda_{\omega^{\prime \prime} j}-s+i+j-n .
\end{aligned}
$$

Since we are assuming that either $n=2 p+1$ or that $i \neq p$, we have $s \geq 0$. Moreover, $\Lambda_{\omega^{\prime \prime} j} \geq 0$, except when $n=2 p$ and $j=p$. In the latter situation we have $\left|\Lambda_{\omega^{\prime \prime} j}\right| \leq s$. Hence $q_{\omega^{\prime \prime} \omega^{\prime}}<0$.

In the second case we find that $\Lambda_{\omega^{\prime \prime} j}=\Lambda_{\omega^{\prime} j}+1$. If $j \neq i$ we have $\lambda+\Lambda_{\omega^{\prime} j}-j$ $+1 \neq 0$, by the uniqueness of the index $i$ in the hypothesis of the theorem. If $j=i$, we have $\Lambda_{\omega^{\prime \prime} i} \leq s$ by the definition of the set $D_{\lambda}^{-}$. Hence $\Lambda_{\omega^{\prime} i}<s$, and $q_{\omega^{\prime \prime} \omega^{\prime}}=\lambda+\Lambda_{\omega^{\prime} i}-i+1<\lambda+s-i+1=0$.

If case three applies, we have $\lambda-p=-s+i-1-p<0$.

Hence, in all three cases, $q_{\omega^{\prime} \omega^{\prime}} \neq 0$. Thus

$$
E_{\omega^{\prime \prime}} \phi_{Y} E_{\omega^{\prime}} g^{\prime}=\left(1 / q_{\omega^{\prime \prime} \omega^{\prime}}\right) E_{\omega^{\prime \prime}} d R(Y) E_{\omega^{\prime}} g^{\prime} \in d R\left(U\left(\mathbf{P}_{\mathbf{C}}(n)\right)\right) \rightarrow,
$$

whenever $E_{\omega^{\prime \prime}} d R(Y) E_{\omega^{\prime}} \neq 0$. Thus $D_{\lambda}^{-} \phi_{Y} D_{\lambda}^{-} g^{\prime} \in d R\left(U\left(\mathbf{P}_{\mathbf{C}}(n)\right)\right) \rightarrow$. Thus, the $\mathbf{G}(n)$-module generated by $\Im$ is an $\delta_{D_{\lambda}^{-}}$-module, and the irreducibility follows.

Finally, we turn to the last assertion of the theorem. If the set $D_{\lambda}^{-}$is equal to the set of all $\mu$-admissible classes, $\Omega_{\mu}(\hat{K}(n))$, then $D_{\lambda}^{-}=1$, and the last assertion is vacuously true. Hence, assume that $1-D_{\lambda}^{-} \neq 0$; then there exists a class $\left[\omega^{\prime}\right] \notin D_{\lambda}^{-}$, and $E_{\omega^{\prime}}\left(1-D_{\lambda}^{-}\right) E_{\omega^{\prime}}=\left(1-D_{\lambda}^{-}\right) E_{\omega^{\prime}}$. We also have for the index $i$ the following inequality $\Lambda_{\omega^{\prime} i}>s$. Let $\left[\omega^{\prime \prime}\right]$ be the class corresponding to the highest weight $\Lambda_{\omega^{\prime}}-\varepsilon_{i}$. (The latter is in fact a dominant integral form because of the inequalities (7) and (6).) Then for all $Y \in \mathbf{P}_{\mathbf{C}}(n)$,

$$
E_{\omega^{\prime \prime}} d R(Y) E_{\omega^{\prime}}=q_{\omega^{\prime \prime} \omega^{\prime}} E_{\omega^{\prime \prime}} \phi_{Y} E_{\omega^{\prime}}
$$

with

$$
\begin{aligned}
q_{\omega^{\prime \prime} \omega^{\prime}} & =\lambda-\Lambda_{\omega^{\prime} i}-n+i+1<\lambda-s-n+i+1 \\
& =-2 s+2 i-n \leq 0 .
\end{aligned}
$$

Hence, by the remark made at the end of the last section, the class [ $\left.\omega^{\prime \prime}\right]$ also lies in the $U(\mathbf{G}(n))$-module generated by $E_{\omega^{\prime}} d C_{\Lambda \mu}(\hat{G}(n))$. Proceeding inductively, we conclude that the latter module contains a class $\left[\omega^{\prime \prime}\right]$ such that $\Lambda_{\omega^{\prime \prime} i} \leq s$. It follows that the latter $U(\mathbf{G}(n))$-module contains a class in the range of $D_{\lambda}^{-}$. In particular, $D_{\lambda}^{-}$has no supplementary invariant projections. Q.E.D. 
Next, we consider a situation which, in a sense to be described later, is dual to the situation described in Theorem 3.

Theorem 4. Fix a real number $\lambda=d \Lambda(H)$. Suppose there exists an index $i$, $1 \leq i \leq p$, and a $\mu$-admissible class $[\omega] \in \Omega_{\mu}(K(n))$ such that $\lambda-\Lambda_{\omega i}-n+i$ $=0$. Assume also that either $i \neq p$, or that $n=2 p+1$. Then the index $i$ defined above is unique. Let $s=\Lambda_{\omega i}$, for the class $[\omega]$ satisfying the above condition. Let $D_{\lambda}^{+}$ be the set defined by

$$
D_{\lambda}^{+}=\left\{\left[\omega^{\prime}\right] \in \Omega_{\mu}(K(n)): \Lambda_{\omega^{\prime} i}>s\right\} .
$$

Then the following statements are true.

(1) The projection $D^{+}=\sum\left\{E_{\omega}:[\omega] \in D_{\lambda}^{+}\right\}$is an invariant projection in $\left[d C_{\Lambda \mu}(\hat{G}(n), \underline{H}), d R\right]$.

(2) The $U(\mathbf{G}(n))$-module $D^{+} d C_{\Lambda \mu}(\hat{G}(n), \underline{H})$ is irreducible.

(3) The projection defined in (1) has no supplementary invariant projections in $d C_{\Lambda \mu}(\hat{G}(n), \underline{H})$. In particular, the irreducible $U(\mathbf{G}(n))$-module defined in (2) is a unique irreducible submodule.

Proof. We state only the details in which this proof differs from the proof of the previous theorem. The statement concerning the uniqueness of the index $i$ follows exactly as in the proof of Theorem 3 .

In order to prove that $D_{\lambda}^{+}$is an invariant projection, we must show that if $\left[\omega^{\prime \prime}\right] \notin D_{\lambda}^{+},\left[\omega^{\prime}\right] \in D_{\lambda}^{+}$, and $\Lambda_{\omega^{\prime \prime}}-\Lambda_{\omega^{\prime}}= \pm \varepsilon_{j}$, then $q_{\omega^{\prime \prime} \omega^{\prime}}=0$. However, for such classes $\left[\omega^{\prime \prime}\right]$ and $\left[\omega^{\prime}\right]$ we must have $j=i, \Lambda_{\omega^{\prime \prime} i} \leq s$, and $\Lambda_{\omega^{\prime} i}>s$. Thus $\Lambda_{\omega^{\prime \prime} i}=s$, and $\Lambda_{\omega^{\prime \prime} i}=\Lambda_{\omega^{\prime} i}-1$. Hence, $q_{\omega^{\prime \prime} \omega^{\prime}}=\lambda-\Lambda_{\omega^{\prime} i}-n+i+1=\lambda-s+i-n=0$, and invariance follows as in the proof of Theorem 3 .

The irreducibility of $D_{\lambda}^{+}$is proved by showing that $q_{\omega^{\prime \prime} \omega^{\prime}} \neq 0$ for $\left[\omega^{\prime \prime}\right],\left[\omega^{\prime}\right]$ $\in D_{\lambda}^{+}$, and $\Lambda_{\omega^{\prime \prime}}-\Lambda_{\omega^{\prime}}=\sigma$, where $\sigma=\varepsilon_{j},-\varepsilon_{j}$, or 0 . The last possibility applies only when $n=2 p+1$.

First assume that $\Lambda_{\omega^{\prime \prime}}-\Lambda_{\omega^{\prime}}=-\varepsilon_{j}$, for $1 \leq j \leq p$. Then $\Lambda_{\omega^{\prime \prime} j}=\Lambda_{\omega^{\prime} j}-1$, and we have $q_{\omega^{\prime \prime} \omega^{\prime}}=\lambda-\Lambda_{\omega^{\prime \prime} i}-n+j+1=\lambda-\Lambda_{\omega^{\prime \prime} j}-n+j$. If $j \neq i$, then the last expression in the above equation is different from zero, by the uniqueness of the index $i$. If $j=i$, then by the definition of $D_{\lambda}^{+}$, we have $q_{\omega^{\prime \prime} \omega^{\prime}}=\lambda-\Lambda_{\omega^{\prime \prime} i}-n+i$ $<\lambda-s-n+i=0$.

Next, assume $\Lambda_{\omega^{\prime \prime}}-\Lambda_{\omega^{\prime}}=\varepsilon_{j}, 1 \leq j \leq p$. Then we have

$$
q_{\omega^{\prime \prime} \omega^{\prime}}=\lambda+\Lambda_{\omega^{\prime} j}+1-j=s+\Lambda_{\omega j}-i-j+n+1 \geq n+1-2 p>0 .
$$

Finally, if $n=2 p+1$ and $\Lambda_{\omega^{\prime \prime}}-\Lambda_{\omega^{\prime}}=0$, then $q_{\omega^{\prime \prime} \omega^{\prime}}=\lambda-p=s+p-i+1$ $\geq 1$.

Hence, in all three cases, $q_{\omega^{\prime \prime} \omega^{\prime}} \neq 0$, and irreducibility follows as in the proof of Theorem 3. 
Finally, we prove the last assertion. Here we must show that if $\left[\omega^{\prime}\right] \notin D_{\lambda}^{+}$, so that we have $\Lambda_{\omega^{\prime} i} \leq s$, we must also have $q_{\omega^{\prime \prime} \omega^{\prime}} \neq 0$, for $\Lambda_{\omega^{\prime \prime}}=\Lambda_{\omega^{\prime}}+\varepsilon_{i}$. However, in this case we have $q_{\omega^{\prime \prime} \omega^{\prime}}=\lambda+\Lambda_{\omega^{\prime} i}+1-i \geq n+1-2 i>0$. The last assertion follows as in Theorem 3. Q.E.D.

We now consider a possibility omitted in both of the last two theorems, namely the situation in which $n=2 p$ and the index $i$ is equal to the rank of $R(n)$, namely $p$. We point out that the theorem about to be stated includes the case when $n=2$ or $\hat{G}(2)=\operatorname{SL}(2, \mathbf{R})$. However, in that case, the inequalities (7) do not apply and the condition of $\mu$-admissibility reduces to the condition that $2 \Lambda_{\omega p}=2 \Lambda_{\omega l}$ is odd if $\mu$ is the nontrivial character of $\hat{M}(2)$, and $2 \Lambda_{\omega 1}$ is even if $\mu$ is the trivial character of $\hat{M}(2)$.

Theorem 5. Assume that $n=2 p$. Fix a real number $\lambda=d \Lambda(H)$. Suppose that there exists a $\mu$-admissible class such that $\Lambda_{\omega p}=s$ satisfies the equation $\lambda+s-p$ $+1=0$. Let $D_{\lambda p}^{+}$and $D_{\lambda p}^{-}$be the subsets of $\Omega_{\mu}(K(n))$ defined by the expressions

$$
\begin{aligned}
& D_{\lambda p}^{-}=\left\{\left[\omega^{\prime}\right] \in \Omega_{\mu}(\hat{K}(n)): \Lambda_{\omega^{\prime} p} \leq s\right\} \\
& D_{\lambda p}^{+}=\left\{\left[\omega^{\prime}\right] \in \Omega_{\mu}(\hat{K}(n)): \Lambda_{\omega^{\prime} p} \geq-s\right\} .
\end{aligned}
$$

In case $s$ is nonnegative, define the subset $D_{\lambda p}^{F}$ as the intersection

$$
D_{\lambda p}^{F}=D_{\lambda p}^{-} \cap D_{\lambda p}^{+}
$$

Let $D_{\lambda p}^{+}, D_{\lambda p}^{-}$, and $D_{\lambda p}^{F}$ be the projections corresponding to the subsets $D_{\lambda p}^{+}, D_{\lambda p}^{-}$, and $D_{\lambda p}^{F}$ as in Lemma 5.

(1) The projections $D_{\lambda p}^{-}, D_{\lambda p}^{+}$, and in case $s \geq 0, D_{\lambda p}^{F}$ are invariant projections.

(2) In case $s \geq 0, D_{\lambda p}^{F}$ is an irreducible projection. If $p=1$, then $D_{\lambda p}^{F}$ is a finitedimensional projection. In case $s<0, D_{\lambda p}^{+}$and $D_{\lambda p}^{-}$are irreducible projections.

(3) In case $s \geq 0, D_{\lambda p}^{F}$ is the unique irreducible projection; that is, $1-D_{\lambda p}^{F}$ contains no supplementary invariant projections. In case $s<0,1-D_{\lambda p}^{+}-D_{\lambda p}^{-}$ contains no invariant projections.

Remark. It follows from inequality (7a) that the conditions $p>1$ and $\Lambda_{\mu p-1}=s$ imply that $D_{\lambda p}^{+}=D_{\lambda p}^{-}=D_{\lambda p}^{F}=1$. In this case, it will follow that $d C_{\Lambda \mu}(\hat{G}(n), \underline{H})$ is irreducible.

Proof. Again the proof follows the same pattern as that of Theorem 3. We emphasize only those details which differ from the proofs of the last two theorems.

If $\left[\omega^{\prime \prime}\right] \notin D_{\lambda p}^{-},\left[\omega^{\prime}\right] \in D_{\lambda p}^{-}$and $\Lambda_{\omega^{\prime \prime}}=\Lambda_{\omega^{\prime}} \pm \varepsilon_{j}$, we must show that $q_{\omega^{\prime \prime} \omega^{\prime}}=0$. However, under the conditions stated, it must follow that $\Lambda_{\omega^{\prime \prime}}=\Lambda_{\omega^{\prime}}+\varepsilon_{p}$, with $\Lambda_{\omega^{\prime \prime} p}=\Lambda_{\omega^{\prime} p}+1$. Hence

$$
q_{\omega^{\prime \prime} \omega^{\prime}}=\lambda+\Lambda_{\omega^{\prime} p}+1-p=\lambda+s+1-p=0
$$


by hypothesis. Hence it follows as in the proof of statement (1) of Theorem 3 that $D_{\lambda p}^{-}$is invariant.

Similarly, if $\left[\omega^{\prime \prime}\right] \notin D_{\lambda p}^{+},\left[\omega^{\prime}\right] \in D_{\lambda p}^{+}$, and $\Lambda_{\omega^{\prime \prime}}-\Lambda_{\omega^{\prime}}= \pm \varepsilon_{j}$, then $\Lambda_{\omega^{\prime \prime}}-\Lambda_{\omega^{\prime}}$ $=-\varepsilon_{p}, \Lambda_{\omega^{\prime \prime} p}=-s-1$, and $\Lambda_{\omega^{\prime} p}=-s$. Hence

$$
q_{\omega^{\prime \prime} \omega^{\prime}}=\lambda-\Lambda_{\omega^{\prime} p}-n+p+1=\lambda+s+1-p=0 .
$$

Hence $D_{\lambda p}^{+}$is invariant.

Finally, we note that if $s \geq 0$, we have $D_{\lambda p}^{F}=D_{\lambda p}^{+} D_{\lambda p}^{-}=D_{\lambda p}^{-} D_{\lambda p}^{+}$. Hence $D_{\lambda p}^{F}$ is an invariant projection.

Next, assume that $s \geq 0$. In order to show that $D_{\lambda p}^{F}$ is irreducible, we show that $q_{\omega^{\prime \prime} \omega^{\prime}} \neq 0$, whenever $\left[\omega^{\prime \prime}\right],\left[\omega^{\prime}\right] \in D_{\lambda p}^{F}$ and $\Lambda_{\omega^{\prime \prime}}-\Lambda_{\omega^{\prime}}= \pm \varepsilon_{j}$ for some $j, 1 \leq j \leq p$. The irreducibility of the projection $D_{\lambda p}^{F}$ will follow as in the proof of part (2) of Theorem 3.

If $\Lambda_{\omega^{\prime \prime}}=\Lambda_{\omega^{\prime}}+\varepsilon_{j}$, then $q_{\omega^{\prime \prime} \omega^{\prime}}=\lambda+\Lambda_{\omega^{\prime} j}+1-j=\left(\Lambda_{\omega^{\prime} j}-s\right)+(p-j)$. If $j=p$, then $\Lambda_{\omega^{\prime} p}=\Lambda_{\omega^{\prime \prime} p}-1 \leq s-1$. Hence $q_{\omega^{\prime \prime} \omega^{\prime}}<0$. If $j<p$, then by the inequalities (7b), $\Lambda_{\omega^{\prime} j} \geq \Lambda_{\mu p-1} \geq s$. Hence $q_{\omega^{\prime \prime} \omega^{\prime}} \geq p-j>0$.

If $\Lambda_{\omega^{\prime \prime}}=\Lambda_{\omega^{\prime}}-\varepsilon_{j}$, then $q_{\omega^{\prime \prime} \omega^{\prime}}=\lambda-\Lambda_{\omega^{\prime} j}-2 p+j+1=-s-\Lambda_{\omega^{\prime} j}-p+j$. If $j<p$, then $\Lambda_{\omega^{\prime} j} \geq \Lambda_{\mu, p-1} \geq s$. Hence, $q_{\omega^{\prime \prime} \omega^{\prime}} \leq-p+j<0$. If $p=j$, then $\Lambda_{\omega^{\prime} p}$ $=\Lambda_{\omega^{\prime \prime} p}+1 \geq-s+1$. Hence $q_{\omega^{\prime \prime} \omega^{\prime}}=-s-\Lambda_{\omega p} \leq-1$. Hence $D_{\lambda p}^{F}$ is irreducible.

Now assume that $s<0$. We will show that $D_{\lambda p}^{+}$is irreducible. Let $\left[\omega^{\prime \prime}\right]$, $\left[\omega^{\prime}\right]$ $\in D_{\lambda p}^{+}$. First assume $\Lambda_{\omega^{\prime \prime}}-\Lambda_{\omega^{\prime}}=\varepsilon_{j}$. Then $q_{\omega^{\prime \prime} \omega^{\prime}}=\Lambda_{\omega^{\prime} j}-s+p-j$, as before. If $j<p$, then $\Lambda_{\omega j} \geq|s|$, by inequalities (7b). Then $q_{\omega^{\prime \prime} \omega^{\prime}} \geq 2|s|+p-j>0$. If $j=p$, then $q_{\omega^{\prime \prime} \omega^{\prime}}=-s+\Lambda_{\omega^{\prime} p} \geq-2 s>0$.

Next assume $\Lambda_{\omega^{\prime \prime}}-\Lambda_{\omega^{\prime}}=-\varepsilon_{j}$. Then $q_{\omega^{\prime \prime} \omega^{\prime}}=-s-\Lambda_{\omega^{\prime} j}-p+j$. If $j<p$, then $\Lambda_{\omega^{\prime} j} \geq-s$, by the inequalities (7b). Then $q_{\omega^{\prime \prime} \omega^{\prime}} \leq-2 s-p+j<0$. If $j=p$, then $\Lambda_{\omega^{\prime \prime} p} \geq-s$ and $\Lambda_{\omega^{\prime} p}=1+\Lambda_{\omega^{\prime \prime} p} \geq-s+1$. Hence, $q_{\omega^{\prime \prime} \omega^{\prime}}=-s-\Lambda_{\omega^{\prime} p} \leq-1$. Hence, the irreducibility of $D_{\lambda p}^{+}$follows as in the proof of part (2) of Theorem 3 .

We continue the assumption that $s<0$. We will show that $D_{\lambda p}^{-}$is irreducible. Let $\left[\omega^{\prime \prime}\right],\left[\omega^{\prime}\right] \in D_{\lambda p}^{+}$. First assume that $\Lambda_{\omega^{\prime \prime}}-\Lambda_{\omega^{\prime}}=\varepsilon_{j}$. Then $q_{\omega^{\prime \prime} \omega^{\prime}}=\Lambda_{\omega^{\prime} j}-s+p$ $-j$. If $j<p$, then $\Lambda_{\omega^{\prime} j} \geq-s$, by the inequalities (7b). Hence, $q_{\omega^{\prime \prime} \omega^{\prime}} \geq p-j>0$. If $j=p, \Lambda_{\omega^{\prime} p}=\Lambda_{\omega^{\prime \prime} p}-1 \leq s-1$. Hence $q_{\omega^{\prime \prime} \omega^{\prime}} \leq-1$. Next, assume $\Lambda_{\omega^{\prime \prime}}-\Lambda_{\omega^{\prime}}$ $=-\varepsilon_{j}$, then $q_{\omega^{\prime \prime} \omega^{\prime}}=-s-\Lambda_{\omega^{\prime} j}-p+j$. If $j=p, q_{\omega^{\prime \prime} \omega^{\prime}}=-s-\Lambda_{\omega^{\prime} p} \geq-2 s>0$. If $j<p$, then $q_{\omega^{\prime \prime} \omega^{\prime}}=-s-\Lambda_{\omega^{\prime} j}-p+j<-s-\Lambda_{\omega j} \leq 0$, since $\Lambda_{\omega j} \geq-s$, by the inequalities (7b). Hence, the projection $D_{\lambda p}^{-}$is irreducible.

We finally prove the last assertion of the theorem. As pointed out in the remark, if $\Lambda_{\mu p-1}=s$, then $D_{\lambda p}^{+}=D_{\lambda p}^{-}=D_{\lambda p}^{F}=d C_{\Lambda \mu}(\hat{G}(n), \underline{H})$. The assertion follows trivially in that case.

We now assume that $\Lambda_{\mu p-1}>|s|$. Assume $s \geq 0$. We show that $D_{\lambda p}^{F}$ has no supplementary submodules. Suppose $\left[\omega^{\prime}\right] \in D_{\lambda p}^{F}$, where $\left[\omega^{\prime}\right]$ is a $\mu$-admissible class, and suppose $\Lambda_{\omega^{\prime} p}<-s$. Then $\Lambda_{\omega^{\prime}}+\varepsilon_{p}$ is the highest weight of a $\mu$ admissible representation class $\left[\omega^{\prime \prime}\right]$, and we have $q_{\omega^{\prime \prime} \omega^{\prime}}=\Lambda_{\omega^{\prime} p}-s<-2 s \leq 0$. Hence it follows by induction, and the remark made at the end of the last section, 
that there exists a "string" of $\mu$-admissible classes whose highest weights form the sequence $\Lambda_{\omega^{\prime}}, \Lambda_{\omega^{\prime}}+\varepsilon_{p}, \ldots, \Lambda_{\omega^{\prime}}+t \varepsilon_{p}$. This string eventually enters $D_{\lambda p}^{F}$. Similarly, if $\left[\omega^{\prime}\right] \notin D_{\lambda p}^{F}$ and $\Lambda_{\omega^{\prime} p}>s$, then $\Lambda_{\omega^{\prime}}-\varepsilon_{p}$ is the highest weight of a $\mu$ admissible representation class $\left[\omega^{\prime \prime}\right]$, and we have $q_{\omega^{\prime \prime} \omega^{\prime}}=-s-\Lambda_{\omega^{\prime} p}<-2 s \leq 0$. Thus there exists a string of $\mu$-admissible classes whose highest weights form the sequence $\Lambda_{\omega^{\prime}}, \Lambda_{\omega^{\prime}}-\varepsilon_{p}, \ldots, \Lambda_{\omega^{\prime}}-t \varepsilon_{p}$. This string eventually enters $D_{\lambda p}^{F}$, again. Hence in both cases, the $U(\mathbf{G}(n))$-module generated by $E_{\omega^{\prime}} d C_{\Lambda \mu}(\hat{G}(n), \underline{H})$ contains the range of $D_{\lambda p}^{F}$.

Now assume that $s<0$. Let $\left[\omega^{\prime}\right]$ be a $\mu$-admissible class not contained in $D_{\lambda p}^{-} \cup D_{\lambda p}^{+}$. Then an entirely similar argument to the one given above shows that the $U(\mathbf{G}(n))$-module generated by the space $E_{\omega^{\prime}} d C_{\Lambda \mu}(\hat{G}(n), \underline{H})$ contains the range of the projection $D_{\lambda p}^{-}+D_{\lambda p}^{+}$. Q.E.D.

9. The eigenvalues of the Casimir operator. In this section we compute the eigenvalues of the Casimir operator $\Omega=\sum_{j=1}^{n} Y_{0 j}^{2}-\sum_{i=1}^{n-1} \sum_{j>i}^{n} X_{i j}^{2}$ for each of the induced representations contemplated in this paper, and observe some simple consequences of this calculation. Here, we write $Y_{0 j}=E_{0 j}+E_{j 0}$, and $X_{i j}=E_{i j}$ $-E_{j i}$, for $1 \leq i<j \leq n$.

First, we make some observations concerning the Casimir operator of the subalgebra $\mathbf{M}(n)$. For $n-2$, of course, this algebra is trivial. For $n=3, \mathbf{M}(n)$ is the abelian Lie algebra $\left\{X_{23}\right\}$, and we define the Casimir operator to be $\Omega_{M}=-X_{23}^{2}$. In case $n>3$, we normalize the Casimir operator in such a manner that $\Omega_{M}=-\sum_{i=2}^{n-1} \sum_{j>i}^{n} X_{i j}^{2}$. Let us pick a Cartan subalgebra of $\mathbf{M}(n), n \geq 3$, which is contained in the Cartan subalgebra of $\mathbf{K}(n)$. We give this Cartan subalgebra an ordering compatible with the ordering in $(-1)^{1 / 2} \mathbf{H}^{K}$. Let $\delta_{M}$ be the linear form on this Cartan subalgebra defined as follows: if $n=3$, we set $\delta_{M}=0$, and if $n>3$, we set $2 \delta_{M}$ equal to the sum of the positive roots of $\mathbf{M}(n)$. Let $\mu$ be an irreducible representation of the subgroup $\hat{M}(n)$, and let $\Lambda_{\mu}$ be its corresponding highest weight. Then it follows from Lemma 2 that the eigenvalue of $\Omega_{M}$ corresponding to this representation is given by $d \mu\left(\Omega_{M}\right)=\left[\left(\Lambda_{\mu}+\delta_{M}, \Lambda_{\mu}+\delta_{M}\right)\right.$ $\left.-\left(\delta_{M}, \delta_{M}\right)\right] \cdot 1$, where we use the symbol $($,$) to denote the restriction of the$ inner product on $(-1)^{1 / 2} \mathbf{H}^{K}$ to the Cartan subalgebra of $\mathbf{M}(n)$.

Lemma 6. The representation $\left[d C_{\Lambda \mu}(\hat{G}(n), \underline{H}), d R\right]$ maps the element $\Omega$ onto the scalar $\gamma(\Omega)$ given by $\gamma(\Omega)=\lambda^{2}-\lambda$, when $n=2$, and $\gamma(\Omega)=\lambda^{2}-(n-1) \lambda$ $+\left[\left(\Lambda_{\mu}+\delta_{M}, \Lambda_{\mu}+\delta_{M}\right)-\left(\delta_{M}, \delta_{M}\right)\right]$, for $n \geq 3$.

Proof. For each function $f \in d C_{\Lambda \mu}(\hat{G}(n), \underline{H})$ define a complex valued linear function $\hat{f}$ on $U(\mathbf{G}(n))$ as follows. For $q \in U(\mathbf{G}(n))$ we set $\hat{f}(q)=d R(q) f(e)$. Since the functions in the space $d C_{\Lambda \mu}(\hat{G}(n), \underline{H})$ are real-analytic, it follows that the map $f \rightarrow \hat{f}$ is a one-to-one map of $d C_{\Lambda \mu}(\hat{G}(n), \underline{H})$ into the algebraic complex linear dual of $U(\mathbf{G}(n))$. This map is obviously linear as well. It follows from this definition and the fact that $\Omega$ is in the center of $U(\mathbf{G}(n))$, that $d R(\Omega) f(q)=\hat{f}(q \Omega)$ $=\hat{f}(\Omega q)$, for all $q \in U(\mathbf{G}(n))$ and $f \in d C_{\Lambda \mu}(\hat{G}(n), \underline{H})$. Let $I$ be the right ideal in 
$U(\mathbf{G}(n))$ generated by the element $\lambda-H$ and the Lie subalgebra $\mathbf{N}(n)$. Then it is clear that if $f \in d C_{\Lambda \mu}(\hat{G}(n), \underline{H})$, the linear function $\hat{f}$ vanishes on this ideal. Next, we take note of the following computation: (Note: $H=Y_{01}$.)

$$
\begin{aligned}
\Omega & =\sum_{j=1}^{n} Y_{0 j}^{2}-\sum_{i=1}^{n-1} \sum_{j>i}^{n} X_{i j}^{2} \\
& =H^{2}-\sum_{j=2}^{n} X_{1 j} Y_{0 j}-\sum_{i=1}^{n-1} \sum_{j>i}^{n} X_{i j}^{2} \quad \operatorname{Mod} I \\
& =H^{2}-(n-1) H-\sum_{j=2}^{n} Y_{0 j} X_{1 j}-\sum_{i=1}^{n-1} \sum_{j>i}^{n} X_{i j}^{2} \\
& =\lambda^{2}-(n-1) \lambda+\sum_{j=2}^{n} X_{i j}^{2}-\sum_{i=1}^{n-1} \sum_{j>i}^{n} X_{i j}^{2} \quad \operatorname{Mod} I \\
& =\lambda^{2}-(n-1) \lambda \quad \text { if } n=2, \\
& =\lambda^{2}-(n-1) \lambda+\Omega_{M} \quad \text { if } n \geq 3 .
\end{aligned}
$$

Hence, for all $f \in d C_{\Lambda \mu}(\hat{G}(n), \underline{H})$, and $q \in U(\mathbf{G}(n))$, we have

$$
\hat{f}(\Omega q)=\left[\lambda^{2}-(n-1) \lambda+d \mu\left(\Omega_{M}\right)\right] \hat{f}(q) .
$$

The lemma then follows from the remarks made above. Q.E.D.

10. On spacially dual representations. We notice that the eigenvalues of the Casimir operator $\Omega$ are invariant under the Weyl reflection $\lambda \rightarrow-\lambda+(n-1)$. We also note that, for a fixed index $i$ and integer $s$, the conditions on $\lambda$ expressed in Theorems 3 and 4 "transform" into each other under this reflection. In this section we shall explain this phenomenon in terms of an interesting duality between representations corresponding to pairs of parameters $\lambda$ that are Weyl images of each other.

The next lemma is essentially known and depends only on the fact that the group $\hat{G}(n)$ has an Iwasawa decomposition. We say that a real connected Lie group $G$ has an Iwasawa decomposition if there exists a maximal compact subgroup $K$ and a maximal solvable subgroup $S$ such that $G=S K$ and $S \cap K=\{e\}$. This property of $G$ insures the existence of an analytic projection $\sigma$ of $G$ onto $S$ such that $g=\sigma(g) \kappa(g)$, for all $g \in G$. For each $g \in G$ we define an analytic diffeomorphism of $K$ onto itself by the formula $\kappa_{g}(k)=\kappa(k g)$, for all $k \in K$. It is a straightforward matter to check that this map is one-to-one and onto and that, in fact, we have $\kappa_{g}(\cdot)^{-1}=\kappa_{g^{-1}}(\cdot)$ for all $g \in G$. For each $g \in G$ the map $\kappa_{g}$ transforms the Haar measure $d k$ on $K$ into an equivalent measure $d\left(\kappa_{g}(k)\right)$. We shall need the Radon-Nikodym derivative corresponding to this transformation of measures.

Lemma 7. Assume that $G$ is unimodular. Then the Radon-Nikodym derivative $d \kappa_{g}(k) / d k$ is given by $d \kappa_{g}(k) / d k=P^{2}(\sigma(k g))$, for $g \in G$, and $k \in K$, where $P^{2}$ is 
the character on $S$ given by $P^{2}(s)=\operatorname{det}\left[\operatorname{Ad}_{S}(s)\right]$. In case $S=\hat{S}(n)$, we have, of course,

$$
d P^{2}(Z)=2 P(Z)= \begin{cases}0, & \text { when } Z \in \mathbf{N}(n) \\ (n-1), & \text { when } Z=Y_{1}\end{cases}
$$

Proof. It is well known that the Radon-Nikodym derivative $d \kappa_{g}(k) / d k$ is given by the Jacobian of the diffeomorphism $\kappa_{g}(k)$. The latter may be computed as follows. For each $C^{\infty}$-function on $K$, it is convenient to extend this function to a $C^{\infty}$-function on $G$ by the extension $\tilde{f}(g)=f(\kappa(g)), g \in G$. For each point $k \in K$, and $Z \in G$ we get a linear mapping of $Z$ into the tangent space at $k$ by the map $Z \rightarrow d L(Z) \tilde{f}(k)=d L\left(P_{K} Z\right) \tilde{f}(k)$, where $P_{K}$ is the projection of $G$ onto $K$ corresponding to the direct sum decomposition $\mathbf{G}=\mathbf{S}+\mathbf{K}$. Let $Z \in K$. Then we have for $f \in C^{\infty}(K)$,

$$
\begin{aligned}
d \kappa_{g}(d L(Z)) f(k) & =\left.(d / d t) f(\kappa(\exp (t Z) k) g)\right|_{t=0} \\
& =\left.(d / d t) \tilde{f}(\exp (t Z) k g)\right|_{t=0}=\left.(d / d t) \tilde{f}(\exp (t Z) \sigma(k g) \kappa(k g))\right|_{t=0} \\
& =\left.(d / d t) \tilde{f}\left(\exp \left(t \operatorname{Ad}\left(\sigma(k g)^{-1}\right) Z\right) \kappa(k g)\right)\right|_{t=0} \\
& =d L\left(P_{K} \operatorname{Ad}\left(\sigma(k g)^{-1} Z\right)\right) f(\kappa(k g)) .
\end{aligned}
$$

Hence $d \kappa_{g}(k) / d k=\operatorname{det}\left(P_{K} \operatorname{Ad}\left(\sigma(k g)^{-1}\right)\right)$. Now, let $s$ be an element of $S$. Then in a basis of $G$ compatible with the direct $\operatorname{sum} \mathbf{G}=\mathbf{S}+\mathbf{K}$, the matrix representation of the linear map Ad $s$ has the following block form:

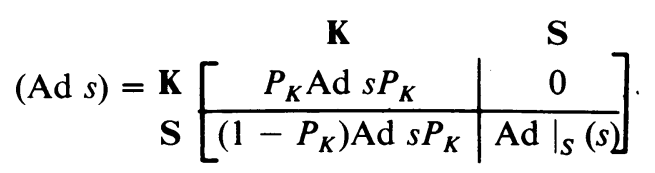

By the fact that $G$ is unimodular, and the above observation we have

$$
1=\operatorname{det}[\operatorname{Ad}(\sigma(k g))]=\operatorname{det}\left[\left.\operatorname{Ad}\right|_{S}(\sigma(k g))\right] \operatorname{det}\left[P_{K} \operatorname{Ad}(\sigma(k g))\right]
$$

Hence $\operatorname{det}\left[P_{K} \operatorname{Ad}\left(\sigma(k g)^{-1}\right)\right]=P^{2}(\sigma(k g))$. Q.E.D.

In order to state the next lemma, it is convenient to view our representation spaces as spaces of functions defined over $\hat{K}(n)$, rather than functions defined on $\hat{G}(n)$. Let $\Lambda$ be a character of the subgroup $\hat{S}(n)$. Let $\tau_{\Lambda}$ denote the restriction-to$\hat{K}(n)$ map taking functions in $C_{\Lambda \mu}(\hat{G}(n), \underline{H})$ onto their restrictions to $\hat{K}(n)$. As pointed out in $\S 2, \tau_{\Lambda}$ is a linear isomorphism of the space $C_{\Lambda \mu}(\hat{G}(n), \underline{H})$ onto the space $C_{\mu}(\hat{K}(n), \underline{H})$. Clearly, this map extends to a unitary equivalence from 
$L_{\Lambda \mu}^{2}(\hat{G}(n), \underline{H})$ onto $L_{\mu}^{2}(\hat{K}(n), \underline{H})$, the space of square-integrable function classes which satisfy condition (2) in $\$ 2$. We also denote this extension by the same symbol $\tau_{\Lambda}$, and define a $\hat{G}(n)$-action on this Hilbert space by the formula $\Pi_{\Lambda}(g)=\tau_{\Lambda} R(g) \tau_{\Lambda}^{-1}$ where $g \in \hat{G}(n)$. We point out that this definition of the group action is independent of the class $[\mu]$ of the representation $[\underline{H}, \mu]$. In fact, let $\phi \in L_{\mu}^{2}(\hat{K}(n), \underline{H})$. Then the explicit expression for this action is as follows: $\Pi_{\Lambda}(g) \phi(k)=\Lambda(\sigma(k g)) \phi(\kappa(k g))$, for all $g \in \hat{G}(n)$, and $k \in \hat{K}(n)$.

If $A$ is a linear operator on $L_{\mu}^{2}(\hat{K}(n), \underline{H})$, we denote by $\bar{A}$ its complex conjugate defined by $\bar{A} \phi=\overline{A \bar{\phi}}$, where $\phi \in L_{\mu}^{2}(\hat{K}(n), \underline{H})$, and the map $\phi \rightarrow \bar{\phi}$ denotes complex conjugation of functions.

We remark incidentally, that complex conjugation of functions takes the space $L_{\mu}^{2}(\hat{K}(n), \underline{H})$ onto the space $L_{\hat{\mu}}^{2}(\hat{K}(n), \underline{H})$, where for any irreducible representation $[\underline{H}, \mu]$ of the subgroup $\hat{M}(n), \bar{\mu}$ denotes the complex conjugate representation of $\mu$. In case $n=2 p$, we have $\mu=\bar{\mu}$, and in case $n=2 p+1$, the complex conjugate representation $\bar{\mu}$ has the property that $\Lambda_{\mu p}=-\Lambda_{\bar{\mu} p}$, and all other components of $\Lambda_{\mu}$ and $\Lambda_{\bar{\mu}}$ with corresponding indices are equal.

Let $A$ be a linear operator on $L_{\mu}^{2}(\hat{K}(n), \underline{H})$. We denote by $A^{*}$ its Hermitian conjugate and by $A^{t}$ its transpose given by $A^{t}=A^{*}$. If $\Pi$ is a representation of $\hat{G}(n)$ on this Hilbert space, we denote by $\widetilde{\Pi}$ its contragredient representation, defined by $\widetilde{\Pi}(g)=\Pi\left(g^{-1}\right)^{t}$, for all $g \in \hat{G}(n)$. We also denote by $\bar{\Pi}$, and $\Pi^{\dagger}$ its complex conjugate and Hermitian conjugate representations respectively. The latter may be defined by the formulas $\bar{\Pi}(g)=\overline{\Pi(g)}$, and $\Pi^{\dagger}(g)=\Pi\left(g^{-1}\right)^{*}$, for all $g \in \hat{G}(n)$. In the case of induced representations we have the following relationships:

Lemma 8. Let $[\underline{H}, \mu]$ be an irreducible representation of $\hat{M}(n)$. Let $\Lambda$ be a character of the subgroup $S(n)$. Then we have the following equalities of group actions on $L_{\mu}^{2}(\hat{K}(n), \underline{H}): \bar{\Pi}_{\Lambda}=\Pi_{\bar{\Lambda}}, \Pi_{\Lambda}=\Pi_{\Lambda^{-1} p^{2}}$, and $\Pi_{\Lambda}^{\dagger}=\Pi_{\Lambda^{-1} p^{2}}$.

Proof. Let $g$ be an arbitrary element of $\hat{G}(n)$, and let $k$ be an arbitrary element of $\hat{K}(n)$. Then for $\phi \in L_{\mu}^{2}(\hat{K}(n), \underline{H})$ we have

$$
\bar{\Pi}_{\Lambda}(g) \phi(k)=\overline{\Pi_{\Lambda}(g) \bar{\phi}(k)}=\bar{\Lambda}(\sigma(k g)) \phi(\kappa(k g))=\Pi_{\bar{\Lambda}}(g) \phi(k) .
$$

In order to check the second relation, let $\psi$ be another element of $L_{\mu}^{2}(\hat{K}(n), \underline{H})$. Then we have

$$
\left\langle\Pi_{\Lambda}(g) \phi, \psi\right\rangle=\int(\bar{\psi}(k), \bar{\Lambda}(\sigma(k g)) \bar{\phi}(\kappa(k g))) d k
$$

By Lemma 7, we express this integral in the terms of the measure $d \kappa(k g)$, and obtain

$$
\left\langle\Pi_{\Lambda}(g) \phi, \psi\right\rangle=\int\left(\bar{\psi}(k), \bar{\Lambda} P^{-2}(\sigma(k g)) \bar{\phi}(\kappa(k g))\right) d \kappa(k g)
$$


Next, change the variable of integration as follows: $k_{1}=\kappa(k g), k=\kappa\left(k_{1} g^{-1}\right)$. We also have

$$
k_{1}=k_{1} g^{-1} g=\sigma\left(k_{1} g^{-1}\right) \kappa\left(k_{1} g^{-1}\right) g .
$$

Hence $\sigma\left(\kappa\left(k_{1} g^{-1}\right) g\right)=\sigma\left(k_{1} g^{-1}\right)^{-1}$. Hence, the integral is equal to

$$
\int\left(\phi\left(k_{1}\right), \bar{\Lambda}^{-1} P^{2}\left(\sigma\left(k_{1} g^{-1}\right)\right) \psi\left(\kappa\left(k_{1} g^{-1}\right)\right)\right) d k_{1}=\left\langle\phi, \Pi_{\bar{\Lambda}^{-1} P^{2}}\left(g^{-1}\right) \psi\right\rangle .
$$

Hence, the third relation follows from the definition of conjugation. The second relation is obvious, by the first and third relations. Q.E.D.

Remark 1. The proof of Lemma 8 depends only on the hypothesis of Lemma 7.

Remark 2. It is clear that the eigenvalues of operators $d \Pi_{\Lambda}(\Omega)$ and $d \Pi_{\Lambda}(\Omega)$ must be equal on the dense subspace $d C_{\mu}(\hat{K}(n), H)$. It follows from Lemma 8 above that this eigenvalue must be invariant under the reflection $d \Lambda \rightarrow d\left(\Lambda^{-1} P^{2}\right)$ $=2 P-d \Lambda$. If one applies these differential characters to the element $H$, one obtains the transformation $\lambda \rightarrow n-1-\lambda$ a fact which explains the symmetry of the eigenvalues of $\Omega$. We remark further that the above argument applies to an arbitrary element $\Omega^{\prime}$ of the center of $U(G(n))$. Thus the eigenvalue of $\Omega^{\prime}$ must be a polynomial in $\lambda$ which is invariant under the Weyl reflection $\lambda \rightarrow n-1-\lambda$. In the case of infinitesimal characters of class 1 representations this fact is a special case of a result of Harish-Chandra (see [10, p. 431]).

We close this section by making some preliminary remarks concerning quotient representations and their relationship to contragredient representations. Let $\Lambda$ be a character on $\hat{S}(n)$. Suppose $D$ is a proper invariant projection in the representation $\left[L_{\mu}^{2}(\hat{K}(n), \underline{H}), \Pi_{\Lambda}\right]$. By Theorem 2 , we know that for this to be the case, the number $\lambda=d \Lambda(H)$ must be an integer or half odd integer. In particular, the character $\Lambda$ must be real. Hence, the Hilbert space adjoint and the contragredient representations coincide in this case.

By the remark made prior to the statement of Lemma 8, as well as by the conditions for proper invariant subspaces in Theorems 3,4 and 5, we know that $D$ is also an invariant projection on the module $\left[L_{\mu}^{2}(\mathcal{K}(n), \underline{H}), \Pi_{\Lambda}\right]$. We also know that $1-D$ is not an invariant projection in this representation. However, one may define a quotient representation on the range of $1-D$ by means of the action $g \rightarrow(1-D) \Pi_{\Lambda}(g)(1-D)$. (The fact that this action defines a representation is a straightforward matter to check.) The name quotient is justified in this context by noting that this representation is isomorphic to the quotient structure $\left[L_{\mu}^{2}(\hat{K}(n), \underline{H}), \Pi_{\Lambda}\right] /\left[D L_{\mu}^{2}(\hat{K}(n), \underline{H}), \Pi_{\Lambda}\right]$. We also note that the $U(\mathbf{G}(n))$-module of $\hat{K}(n)$-finite vectors in the representation $\left[(1-D) L_{\mu}^{2}(\hat{R}(n), \underline{H}),(1-D) \Pi_{\Lambda}(\cdot)(1\right.$ - D)] is isomorphic to the quotient module $\left[d C_{\mu}(\hat{K}(n), \underline{H}), d \Pi_{\Lambda}\right] /\left[D d C_{\mu}(\hat{K}(n), \underline{H})\right.$, $\left.d \Pi_{\Lambda}\right]$. We assert that the representation $\Pi_{\Lambda}=\Pi_{\Lambda^{-1} P^{2}}$ also acts on the spaces $(1-D) L_{\mu}^{2}(\hat{K}(n), \underline{H})$ and $(1-D) L_{\hat{\mu}}^{2}(\hat{K}(n), \underline{H})$. This last assertion is established by noting that for all $\psi, \phi \in L_{\mu}^{2}(\hat{K}(n), \underline{H})$, and $g \in \hat{G}(n)$, we have 


$$
0=\left\langle\Pi_{\Lambda}\left(g^{-1}\right)(1-D) \phi, D \psi\right\rangle=\left\langle(1-D) \phi, \Pi_{\Lambda}(g) D \psi\right\rangle=0,
$$

since $D$ is invariant under the operator $\Pi_{\Lambda}(g)$. Hence, $\Pi_{\Lambda}\left(g^{-1}\right)(1-D)$ lies in the range of $D^{\perp}=(1-D)$, and the assertion is proved. In the next section we will show the quotient action defined above is infinitesimally equivalent to the representation $\left[(1-D) L_{\hat{\mu}}^{2}(\hat{K}(n), \underline{H}), \tilde{\Pi}_{\Lambda^{-1} P^{2}}\right]$. At this point we point out the assertion just established "explains" the relationship between the condition on $\lambda$ expressed by the hypothesis of Theorem 3 and that of Theorem 4.

12. The classification of quasi-simple irreducible representations of $\hat{G}(n)$. We will now show how our methods, together with some general results of HarishChandra, lead to a complete classification, up to infinitesimal equivalence, of irreducible quasi-simple representations of $\hat{G}(n)$. As pointed out in the introduction, the latter classification is the same as the classification, up to Naimark equivalence, of the topologically completely irreducible representations of $\hat{G}(n)$. We summarize the main conclusions of this paper in the following theorem.

Theorem 6. Let $[\underline{H}, \mu]$ be an irreducible representation of $\hat{M}(n)$, and let $\Lambda$ be a character of the subgroup $\hat{S}(n)$. Let $\lambda$ be defined by $\lambda=d \Lambda(H)$. Then the following statements are true:

(1) A necessary and sufficient condition that the $U(\mathbf{G}(n))$-module $\left[d C_{\mu}(\hat{K}(n), \underline{H})\right.$, $\left.d \Pi_{\Lambda}\right]$ be irreducible is that one of the following statements be true:

(a) For all $\mu$-admissible classes [ $\omega]$ we have $\lambda+\Lambda_{\omega i}+1-i \neq 0$, and $\lambda-\Lambda_{\omega i}$ $-n+i+1 \neq 0$.

(b) For some $\mu$-admissible class $[\omega]$ we have $\lambda+\Lambda_{\omega i}+1-i=0, i>1$, and $\Lambda_{\omega i}=\Lambda_{\mu i-1}$, or $\lambda-\Lambda_{\omega i}-n+i+1=0, i \leq p$, and $\Lambda_{\omega i}=\Lambda_{\mu i}$.

(2) Assume that the $U(\mathbf{G}(n))$-module $\left[d C_{\mu}(\hat{K}(n), \underline{H}), d \Pi_{\Lambda}\right]$ is irreducible. Then a necessary and sufficient condition for this module to be equivalent to a $U(\mathbf{G}(n))$ module $\left[d C_{\mu^{\prime}}(\hat{K}(n), \underline{H}), d \Pi_{\Lambda^{\prime}}\right]$ is that $\mu^{\prime}=\mu$ and $\Lambda^{\prime}=\Lambda$, or $\mu^{\prime}=\bar{\mu}$ and $\Lambda^{\prime}=\Lambda^{-1} P^{2}$.

(3) Assume that there exists a $\mu$-admissible class [ $\omega]$ and an index $i, 1 \leq i \leq p$, such that $\lambda+\Lambda_{\omega i}-n+i=0$. Let $s$ be the integer or half odd integer such that $\lambda+s-n+i=0$. Assume also that $i \neq p$ or that $n=2 p+1$, and that $s \neq \Lambda_{\mu i-1}$, if $i>1$. Let $D_{\lambda}^{+}$denote the set of $\mu$-admissible classes $\left[\omega^{\prime}\right]$ whose highest weights $\Lambda_{\omega^{\prime}}$ satisfy the condition $\Lambda_{\omega^{\prime} i}>$ s. Let $D_{\lambda}^{+}$denote the projection defined by

$$
D_{\lambda}^{+}=\sum\left\{E_{\omega}:[\omega] \in D_{\lambda}^{+}\right\}
$$

Then the following statements are true:

(a) $\left[D_{\lambda}^{+} d C_{\mu}(\hat{K}(n), \underline{H}), d \Pi_{\Lambda}\right]$ is an irreducible proper submodule of the $U(\mathbf{G}(n))$ module $\left[d C_{\mu}(\hat{K}(n), \underline{H}), d \Pi_{\Lambda}\right]$.

(b) $\left[D_{\lambda}^{+} d C_{\bar{\mu}}(\hat{K}(n), \underline{H}), d \Pi_{\Lambda}\right]$ is an irreducible proper submodule of the $U(\mathbf{G}(n))$ module $\left[d C_{\bar{\mu}}(\hat{K}(n), \underline{H}), d \Pi_{\Lambda}\right]$.

(c) Let $\lambda^{\prime}=-\lambda+n-1$. In the notation of Theorems 3 and 4 we have $D_{\lambda^{\prime}}^{-}$ 
$=1-D_{\lambda}^{+}$, and the space $\left[D_{\lambda^{\prime}}^{-} d C_{\mu}(\hat{K}(n), \underline{H}), d \Pi_{\Lambda^{-1} P^{2}}\right]$ is a proper irreducible submodule of $\left[d C_{\mu}(\hat{K}(n), \underline{H}) d \Pi_{\Lambda^{-1} P^{2}}\right]$.

(d) $\left[D_{\lambda^{\prime}}^{-} d C_{\bar{\mu}}(R(n), \underline{H}), d \Pi_{\Lambda^{-1} P^{2}}\right]$ is a proper irreducible submodule of $\left[d C_{\bar{\mu}}(\hat{K}(n), \underline{H})\right.$, $\left.d \Pi_{\Lambda^{-1} P^{2}}\right]$.

(e) We have the following isomorphism of $U(\mathbf{G}(n))$-modules:

$$
\begin{aligned}
& {\left[D_{\lambda}^{+} d C_{\mu}(\hat{K}(n), \underline{H}), d \Pi_{\Lambda}\right]} \\
& \cong\left[d C_{\bar{\mu}}(\hat{K}(n), \underline{H}), d \Pi_{\Lambda^{-1} P^{2}}\right] /\left[D_{\lambda^{\prime}}^{-} d C_{\bar{\mu}}(\hat{K}(n), \underline{H}) d \Pi_{\Lambda^{-1} P^{2}}\right], \\
& {\left[D_{\lambda^{\prime}}^{-} d C_{\mu}(\hat{K}(n), \underline{H}), d \Pi_{\Lambda^{-1} p^{2}}\right]} \\
& \cong\left[d C_{\bar{\mu}}(\hat{K}(n), \underline{H}), d \Pi_{\Lambda}\right] /\left[D_{\lambda}^{+} d C_{\bar{\mu}}(R(n), \underline{H}), d \Pi_{\Lambda}\right] .
\end{aligned}
$$

Of course, we have a similar pair of isomorphisms by interchanging $\mu$ and $\bar{\mu}$.

(f) If $n=2 p$, the isomorphisms listed in (e) are the only isomorphisms of $U(\mathbf{G}(n))$ modules occuring in this case.

(g) If the index $i=1$, then $\left[D_{\lambda}^{-} d C_{\mu}(\hat{K}(n), \underline{H}), d \Pi_{\Lambda^{-1} P^{2}}\right]$ is an irreducible finitedimensional $U(\mathbf{G}(n))$-module.

(4) Now assume that $n=2 p$ and assume that there exists a $\mu$-admissible class $[\omega]$ such that the number $\Lambda_{\omega p}=s$ satisfies the equation $\lambda+s-p+1=0$. Assume also that $s<0$. Then $\lambda^{\prime}=-\lambda+n-1$ satisfies the equation $\lambda^{\prime}+s^{\prime}-p+1=0$, with $s^{\prime}=-(s+1)$. Hence, we may define the projections $D_{\lambda p}^{+}, D_{\lambda p}^{-}$, and $D_{\lambda^{\prime} p}^{F}$ as in the statements of Theorem 5. Then the following are irreducible $U(\mathbf{G}(n))$-modules: $\left[D_{\lambda p}^{-} d C_{\mu}(\hat{K}(n), \underline{H}), d \Pi_{\Lambda}\right],\left[D_{\lambda p}^{+} d C_{\mu}(\hat{K}(n), \underline{H}), d \Pi_{\Lambda}\right]$, and $\left[D_{\lambda^{\prime} p}^{F} d C_{\mu}(\hat{K}(n), \underline{H}), d \Pi_{\Lambda^{-1} P^{2}}\right]$. We also have the following equivalence of $U(G(n))$-modules:

$$
\begin{aligned}
& {\left[D_{\lambda^{\prime} p}^{F} d C_{\mu}(\hat{K}(n), \underline{H}), d \Pi_{\Lambda^{-1} P^{2}}\right]} \\
& \quad \cong\left[d C_{\mu}(\hat{K}(n), \underline{H}) d \Pi_{\Lambda}\right] /\left[\left(D_{\lambda p}^{-}+D_{\lambda p}^{+}\right) d C_{\mu}(\hat{K}(n), \underline{H}), d \Pi_{\Lambda}\right] \\
& {\left[D_{\lambda p}^{-} d C_{\mu}(\hat{K}(n), \underline{H}), d \Pi_{\Lambda}\right] \oplus\left[D_{\lambda p}^{+} d C_{\mu}(\hat{K}(n), \underline{H}), d \Pi_{\Lambda}\right]} \\
& \quad \cong\left[d C_{\mu}(\hat{K}(n), \underline{H}), d \Pi_{\Lambda^{-1} P^{2}}\right] /\left[D_{\lambda^{\prime} p}^{F} d C_{\mu}(\hat{K}(n), \underline{H}), d \Pi_{\Lambda^{-1} P^{2}}\right] .
\end{aligned}
$$

These are the only $U(\mathbf{G}(n))$-module isomorphisms occuring in this case.

(5) Let $\left[H_{\Pi}, \Pi\right]$ be a strongly continuous representation of $\hat{G}(n)$ on a Banach space $H_{\Pi}$. Assume that $\left[H_{\Pi}, \Pi\right]$ is irreducible and quasi-simple, or topologically completely irreducible. Let $\left[d H_{\Pi}, d \Pi\right]$ denote the $U(\mathbf{G}(n))$-module of $\hat{K}(n)$-finite vectors in this representation. Then there exists a finite-dimensional irreducible representation $[\underline{H}, \Lambda \mu]$ of the subgroup $\hat{S}(n) \hat{M}(n)$ such that precisely one of the following statements is true:

(a) The character $\Lambda$ satisfies conditions (a) or (b) of statement (1) and we have the following isomorphisms of $U(\mathbf{G}(n))$-modules: 


$$
\begin{aligned}
{\left[d H_{\Pi}, d \Pi\right] } & \cong\left[d C_{\mu}(\hat{K}(n), \underline{H}), d \Pi_{\Lambda}\right] \\
& \cong\left[d C_{\mu}(\hat{K}(n), \underline{H}), d \Pi_{\Lambda^{-1} P^{2}}\right]
\end{aligned}
$$

(b) The character $\Lambda$ satisfies the hypothesis of statement (3) and we have precisely one of the following two possible $U(\mathbf{G}(n))$-module isomorphisms:

$$
\left[d H_{\Pi}, d \Pi\right] \cong\left[D_{\lambda}^{+} d C_{\mu}(\hat{K}(n), \underline{H}), d \Pi_{\Lambda}\right]
$$

or

$$
\left[d H_{\Pi}, d \Pi\right] \cong\left[D_{\lambda^{\prime}}^{-} d C_{\mu}(\hat{K}(n), \underline{H}) d \Pi_{\Lambda^{-1} P^{2}}\right] .
$$

(c) The character $\Lambda$ satisfies the hypothesis of statement (4), and we have precisely one of the following three $U(\mathbf{G}(n))$-module isomorphisms:

$$
\begin{aligned}
& {\left[d H_{\Pi}, d \Pi\right] \cong\left[D_{\lambda p}^{-} d C_{\mu}(\hat{K}(n), \underline{H}), d \Pi_{\Lambda}\right],} \\
& {\left[d H_{\Pi}, d \Pi\right] \cong\left[D_{\lambda p}^{+} d C_{\mu}(\hat{K}(n), \underline{H}), d \Pi_{\Lambda}\right],}
\end{aligned}
$$

or

$$
\left[d H_{\Pi}, d \Pi\right] \cong\left[D_{\lambda^{\prime} p}^{F} d C_{\mu}(\hat{K}(n), \underline{H}), d \Pi_{\Lambda^{-1} p^{2}}\right]
$$

Proof of statement (1). First we show that statement (a) or statement (b) is sufficient for irreducibility. The fact that statement (a) is sufficient is immediate from Theorem 2. If statement (b) is true, then either one of two possibilities occur:

(I) $\lambda+\Lambda_{\omega i}+1-i=0, i>1$, and $\Lambda_{\omega i}=\Lambda_{\mu i-1}$,

(II) $\lambda-\Lambda_{\omega i}-n+i+1=0, i \leq p$, and $\Lambda_{\omega i}=\Lambda_{\mu i}$.

If possibility (I) occurs, we set $s=\Lambda_{\omega i}=\Lambda_{\mu i-1}$. If $i \neq p$, or if $n=2 p+1$, we define the projection $D_{\lambda}^{-}$as in the statement of Theorem 3. By the inequalities (7), every $\mu$-admissible class $\left[\omega^{\prime}\right]$ satisfies the condition that $\Lambda_{\omega i} \leq s$. Thus $D_{\lambda}^{-}=1$, so that irreducibility follows. In case $n=2 p$, and $i=p$, irreducibility follows from the remark made after the statement of Theorem 5 .

If possibility (II) occurs, we set $s=\Lambda_{\omega i}-1=\Lambda_{\mu i}-1$. In this case, every $\mu$ admissible class $\left[\omega^{\prime}\right]$ satisfies the condition that $\Lambda_{\omega i}>s$. Hence the projection $D_{\lambda}^{+}$ defined in the statement of Theorem 4 is equal to the identity. Hence irreducibility follows.

In order to prove that statements (a) or (b) are necessary, we assume that both (a) and (b) are false. Then we have one of the following two possibilities.

(III) $\lambda+\lambda_{\omega}+1-i=0$, and in case $i>1, \Lambda_{\omega i}<\Lambda_{\mu i-1}$.

(IV) $\lambda-\Lambda_{\omega i}-n+i+1=0$, and in case $i \leq p, \Lambda_{\omega i}>\Lambda_{\mu i}$.

If possibility (III) holds, and $i \neq p$, or $n=2 p+1$, the projection $D_{\lambda}^{-}$defined 
in the statement of Theorem 3, is not the identity on $d C_{\mu}(R(n), \underline{H})$. Similarly, in case $i=p$, and $n=2 p$, the projection $D_{\lambda p}^{-}$defined in the statement of Theorem 5 is not the identity; so that $d C_{\mu}(\hat{K}(n), \underline{H})$ contains a proper invariant subspace.

Similarly, if possibility (IV) holds, and $i \neq p$, or $n=2 p+1$, then the projection $D_{\lambda}^{+}$is proper, and hence, by Theorem $4, d C_{\mu}(R(n), \underline{H})$ is not irreducible. If $n=2 p$ and $i=p$, then $d C_{\mu}(\hat{K}(n), \underline{H})$ is not irreducible, by Theorem 5 , because the projection $D_{\lambda p}^{+}$is proper. Hence statement (1) is proved.

For the proof of statement (2), as well as other equivalence statements, use will be made of some results of Harish-Chandra on the characters of the induced representations under consideration.

Let $f$ be a $C^{\infty}$-function of compact support on $\hat{G}(n)$. Let $\Pi_{\Lambda \mu}(f)$ be the operator on the space $L_{\mu}^{2}(\hat{R}(n), \underline{H})$ defined by

$$
\Pi_{\Lambda \mu}(f)=\int_{G(n)} f(g) \Pi_{\Lambda}(g) d g .
$$

As pointed out by Harish-Chandra, in [9c, pp. 241-245], this operator is of trace class. Moreover, let $T_{\Lambda \mu}(f)$ denote the trace of this operator. Then the map $f \rightarrow T_{\Lambda \mu}(f)$ is a distribution on $\hat{G}(n)$, in the sense of Schwartz.

Let $\chi_{\mu}$ denote the character of the representation $\mu$. As pointed out in the last section, when $n=2 p$, this character remains unchanged under complex conjugation. While for $n=2 p+1$, the character $\bar{\chi}_{\mu}=\chi_{\bar{\mu}}$ corresponds to the highest weight $\Lambda_{\bar{\mu}}$ which is related to $\Lambda_{\mu}$ by the equations $\Lambda_{\bar{\mu} i}=\Lambda_{\mu i}, 1 \leq i<p$, and $\Lambda_{\bar{\mu} p}=-\Lambda_{\mu p}$. Let $\mathbf{H}^{-}$be a Cartan subalgebra of $\mathbf{M}(n)$. Then $\mathbf{H}_{0}=\operatorname{span}\left\{\mathbf{H}^{-}, H\right\}$ is a Cartan subalgebra of $\mathbf{G}(n)$. Let $H_{0}$ denote the centralizer in $\hat{G}(n)$ of $\mathbf{H}_{0}$. Let $\hat{f}$ be a representative in $R(n)$ of the nonidentity element in the Weyl group of the symmetric pair $(\hat{G}(n), \hat{K}(n))$. Then $\mathrm{Ad} \hat{f}$ fixes the subalgebra $\mathbf{H}^{-}$, and we have $\chi_{\mu}\left(f m f^{-1}\right)=\chi_{\bar{\mu}}(m)$ for all $m \in \hat{M}(n)$. It follows from Theorem 2 of HarishChandra [9d, p. 511], that the character $T_{\Lambda \mu}(f)$ is given by the following formula

$$
T_{\Lambda \mu}(f)=C \int_{G(n)} f(g) \Theta_{\Lambda \mu}(g) d g
$$

where $C$ is a constant, and where $g \rightarrow \Theta_{\Lambda \mu}(g)$ is a function which is defined almost everywhere on $\hat{G}(n)$, more explicitly, on the set $G_{1}$, consisting of regular elements conjugate to elements in $H_{0}$, by the formulas

$\Theta_{\Lambda \mu}(\operatorname{Ad} g h)=\Theta_{\Lambda \mu}(h)$

$$
\begin{aligned}
=(1 / \sinh t)\left\{\exp \left[\left(\lambda-\frac{(n-1)}{2}\right) t\right] \chi_{\mu}\left(h_{-}\right)\right. & \\
& \left.+\exp \left[\left(-\lambda+\left(\frac{n-1}{2}\right)\right) t\right] \chi_{\bar{\mu}}\left(h_{-}\right)\right\}
\end{aligned}
$$


where $h=\exp (t H) h_{-}, h_{-} \in H_{0} \cap \hat{M}(n)$.

The effect of the nontrivial Weyl reflection on the character $\Lambda$ is the following: $\lambda \rightarrow-\lambda+n-1$. Hence by Lemma 12 of [9d] it follows that two characters $T_{\Lambda \mu}$ and $T_{\Lambda^{\prime} \mu^{\prime}}$ are equal if and only if $\mu=\mu^{\prime}$ and $\Lambda=\Lambda^{\prime}$, or $\mu^{\prime}=\bar{\mu}$ and $\Lambda^{\prime}=\Lambda^{-1} P^{2}$. The last condition is equivalent to the condition that $\lambda^{\prime}=-\lambda+n-1$. By Theorem 6 of [9c, p. 248], it follows that the irreducible $U(\mathbf{G}(n))$-modules $\left[d C_{\mu}(R(n), \underline{H}), d \Pi_{\Lambda}\right]$ and $\left[d C_{\mu^{\prime}}(\hat{K}(n), \underline{H}), d \Pi_{\Lambda^{\prime}}\right]$ are equivalent if and only if $\mu^{\prime}=\mu$ and $\Lambda=\Lambda^{\prime}$; or $\mu=\bar{\mu}$ and $\Lambda^{\prime}=\Lambda^{-1} P^{2}$.

Proof of statement (3). We note that under the hypothesis of statement (3), $D_{\lambda}^{+}$ is a proper nonempty subset of $\Omega_{\mu}(\hat{K}(n))$. Also from the inequalities (7), $D_{\lambda}^{+}$is a proper nonempty subset of $\Omega_{\bar{\mu}}(\hat{K}(n))$. Conclusions (a) and (b) follow immediately from Theorem 4. Let $[\omega]$ be the class such that $\lambda+\Lambda_{\omega i}-n+i=0$. Then we also have $\lambda^{\prime}-\Lambda_{\omega i}-i+1=0$. Hence, the set $D_{\lambda^{\prime}}^{-}$is a proper subset of $\Omega_{\mu}(\hat{K}(n))$ such that $D_{\lambda^{\prime}}^{-} \cup D_{\lambda}^{+}=\Omega_{\mu}(R(n))=\Omega_{\bar{\mu}}(\hat{K}(n))$. Hence, conclusions (c) and (d) follow from Theorem 3. We now turn to the proof of conclusion (e). By Theorem 6 of [9c] it is sufficient to show that for each $C^{\infty}$-function of compact support $f$ the traces of the operators $D_{\lambda}^{+} \Pi_{\Lambda \mu}(f) D_{\lambda}^{+}$and $D_{\lambda}^{+} \Pi_{\Lambda^{-1} p^{2} \bar{\mu}}(f) D_{\lambda}^{+}$are equal, as are the traces of the operators $D_{\lambda^{\prime}}^{-} \Pi_{\Lambda \mu}(f) D_{\lambda^{\prime}}^{-}$and $D_{\lambda^{\prime}}^{-} \Pi_{\Lambda^{-1} P^{2} \bar{\mu}}(f) D_{\lambda^{\prime}}^{-}$. The proof of statement (e) then follows by the discussion at the end of the last section. For each class $[\omega] \in \Omega(R(n))$ we define a $C^{\infty}$-function $E_{\omega} f$ by the formula

$$
\int_{R(n)} \bar{\chi}_{\omega}(k) R(k) f d k=E_{\omega} f
$$

Then, by a straightforward application of the invariance of the Haar integral, and the Schur orthogonality relations we have, for all $\Lambda$ and $\mu, \Pi_{\Lambda \mu}(f) E_{\omega}$ $=\Pi_{\Lambda \mu}\left(E_{\omega} f\right)$. Thus, we have, by the equality of the characters $T_{\Lambda \mu}$ and $T_{\Lambda^{-1} P^{2} \bar{\mu}}$,

$$
\begin{aligned}
\operatorname{trace}\left(E_{\omega} \Pi_{\Lambda \mu}(f) E_{\omega}\right) & =\operatorname{trace}\left[\Pi_{\Lambda \mu}\left(E_{\omega} f\right)\right] \\
& =\operatorname{trace}\left[E_{\omega} \Pi_{\Lambda^{-1} P^{2} \mu}\left(E_{\omega} f\right)\right]=\operatorname{trace}\left[E_{\omega} \Pi_{\Lambda^{-1} P^{2} \mu}(f) E_{\omega}\right] .
\end{aligned}
$$

By the discussion in [9c, p. 242], it follows that the series

$$
\sum\left\{\operatorname{Trace}\left[E_{\omega} \Pi_{\Lambda \mu}(f) E_{\omega}\right]:[\omega] \in \Omega_{\mu}(\hat{K}(n))\right\}
$$

converges absolutely to $T_{\Lambda \mu}(f)$. Hence the series

$$
\sum\left\{\operatorname{Trace}\left[E_{\omega} \Pi_{\Lambda \mu}(f) E_{\omega}\right]:[\omega] \in D_{\lambda}^{+}\right\}
$$

converges absolutely to the trace of $D_{\lambda}^{+} \Pi_{\Lambda \mu}(f) D_{\lambda}^{+}$as well as to the trace of $D_{\lambda}^{+} \Pi_{\Lambda^{-1} P^{2} \mu}(f) D_{\lambda}^{+}$. Similarly, the series

$$
\sum\left\{\operatorname{Trace}\left[E_{\omega} \Pi_{\Lambda \mu}(f) E_{\omega}\right]:[\omega] \in D_{\lambda^{\prime}}^{-}\right\}
$$


converges absolutely to the trace

$$
\operatorname{trace}\left[D_{\lambda^{\prime}}^{-} \Pi_{\Lambda^{-1} P^{2} \mu}(f) D_{\lambda^{\prime}}^{-}\right]=\operatorname{trace}\left[D_{\lambda^{\prime}}^{-} \Pi_{\Lambda \mu}(f) D_{\lambda^{\prime}}^{-}\right]
$$

Finally, the fact that the $U(\mathbf{G}(n))$-module isomorphisms appearing in statement (e) are the only ones possible is deduced from the following argument. Let [ $\omega]$ be a class in the set $D_{\lambda}^{+}$. Then we define for each $C^{\infty}$-function of compact support $f$, defined on the interval $(0, \infty)$, the conjugacy class function $F$ :

$$
F(\operatorname{Ad} g h)=F(h)=f(t) \chi_{\omega}\left(h_{-}\right)
$$

where $g \in \hat{G}(n), h=\exp (t H) h_{-}$, and $h_{-} \in \hat{M}(n) \cap H_{0}$. Then there exists a measure $\Delta_{-}\left(h_{-}\right) d h_{-}$on the $\hat{M}(n)$-conjugacy class of $\hat{M}(n)$ such that

$$
T_{\Lambda \mu}(F)=C^{\prime} \int_{0}^{\infty} f(t)[\sinh t]^{n-1} \int \Theta_{\Lambda \mu}\left(\exp (t H) h_{-}\right) \chi_{\omega}(h) \Delta_{-}\left(h_{-}\right) d h_{-}
$$

where $C^{\prime}$ is a constant different from zero. See [9d, p. 510]. We also note that by the remark made above

$$
\Pi_{\Lambda \mu}(F)=\Pi_{\Lambda \mu}(F) E_{\omega}=\Pi_{\Lambda \mu}(F) D_{\lambda}^{+}
$$

Hence, by (8),

$$
\begin{aligned}
\operatorname{Trace}\left(\Pi_{\Lambda \mu}(F) D_{\lambda}^{+}\right)= & C^{\prime} K_{\omega \mu} \int_{0}^{\infty} f(t) \exp [(\lambda-(n-1) / 2) t] d t \\
& +C^{\prime} K_{\omega \bar{\mu}} \int_{0}^{\infty} f(t) \exp [(-\lambda+(n-1) / 2) t] d t
\end{aligned}
$$

where $K_{\omega \mu}=\bar{K}_{\omega \bar{\mu}} \neq 0$. Since $f$ is an arbitrary $C^{\infty}$-function having compact support, it follows from the linear independence of exponential functions belonging to distinct exponents, that the distribution $F \rightarrow D_{\lambda}^{+} \Pi_{\Lambda \mu}(F) D_{\lambda}^{+}$is equal to the distribution $F \rightarrow D_{\lambda}^{+} \Pi_{\Lambda^{\prime \prime} \mu^{\prime \prime}}(F) D_{\lambda}^{+}$only if $\Lambda=\Lambda^{\prime \prime}$ and $\mu=\mu^{\prime \prime}$, or if $\Lambda^{\prime \prime}=\Lambda^{-1} P^{2}$ and $\mu^{\prime \prime}=\bar{\mu}$. The corresponding statements for the representations $D_{\lambda^{\prime}}^{-} \Pi_{\Lambda \mu} D_{\lambda^{\prime}}^{-}$follow similarly. Hence the uniqueness conclusion follows from the theorem of Harish-Chandra quoted earlier.

Statement (f) is obvious since in case $i=1$, the set $D_{\lambda}^{-}$is finite.

Proof of statement (4). The irreducibility conclusions are a restatement of those in Theorem 5. We note that under hypothesis of the statement we have $1=D_{\lambda p}^{-}+D_{\lambda p}^{+}+D_{\lambda^{\prime} p}^{F}$, and we also note that the projections $D_{\lambda p}^{-}, D_{\lambda p}^{+}$and $D_{\lambda^{\prime} p}^{F}$ are mutually orthogonal. The equivalence conclusions follow from the discussion at the end of the last paragraph and the results of Harish-Chandra quoted above, exactly as in the proof of statements (3e) and (3f).

Proof of statement (5). Let $\left[H_{\Pi}, \Pi\right]$ be a strongly continuous representation of $\hat{G}(n)$ on a Banach space $H_{\Pi}$, and assume that this representation is topologically 
irreducible and quasi-simple. As pointed out in the introduction, these conditions are equivalent to assuming that $\left[H_{\Pi}, \Pi\right]$ is completely irreducible. We note, moreover, that every class in $\Omega(\hat{K}(n))$ occurs in the restriction to $\hat{K}(n)$ of some finite-dimensional representation of $\hat{G}(n)$. Hence, the hypothesis of Theorem 4 of Harish-Chandra [9b] is satisfied. Hence, by that theorem, there exists an irreducible representation $[\underline{H}, \Lambda \mu]$ of the parabolic subgroup $\hat{S}(n) \hat{M}(n)$ and a projection $P$ on the space $L_{\mu}^{2}(\hat{K}(n), \underline{H})$ such that $P$ is an invariant projection in the representation $\left[L_{\mu}^{2}(\hat{K}(n), \underline{H}), \Pi_{\Lambda}\right]$, and the representation $\left[H_{\Pi}, \Pi\right]$ is infinitesimally equivalent to the quotient representation $\left[L_{\mu}^{2}(\hat{K}(n), \underline{H}), \Pi_{\Lambda}\right] /\left[P L_{\mu}^{2}(\hat{K}(n), \underline{H})\right.$, $\Pi_{\Lambda}$ ]. By the previous statements of the theorem, all such quotients are completely classified, up to the infinitesimal equivalence. Hence the theorem follows.

13. Some special cases. In this section we compare some special consequences of Theorem 6 with some results in the literature. For the purposes of these comparisons, it is sometimes convenient to consider, in addition to the character $\Lambda$ which occurs in the hypothesis of Theorem 6, the "inducing" character $\Lambda_{0}=\Lambda P^{-1}$. (See the definition of induced representations given in $\S 2$.) For the corresponding infinitesimal character we write $\lambda_{0}=d \Lambda_{0}(H)$. Thus we have $\lambda_{0}=\lambda-(n-1) / 2$. In terms of this parameter the Weyl reflection $\lambda \rightarrow \lambda^{\prime}$ $=-\lambda+n-1$ becomes $\lambda_{0} \rightarrow-\lambda_{0}$.

A. The case $n=2$. In this case, as was pointed out earlier, $\hat{G}(2)$ is isomorphic to $\operatorname{SL}(2, \mathbf{R})$, and the subgroup $\hat{K}(2)$ is isomorphic to a circle group. The set $\Omega(R(2))$ may be identified with the set of half integers. The space of $\hat{K}(2)$-finite functions on the subgroup $\hat{K}(2)$ is given by the space of trigonometric polynomials

$$
\operatorname{span}_{\mathbf{C}}\left\{e_{m}(\cdot): m \in \Omega(\hat{K}(2))\right\},
$$

where $e_{m}\left(\widehat{\exp }\left(\Theta X_{12}\right)\right)=\exp \left[m\left((-1)^{1 / 2} \Theta\right)\right], 0 \leq \Theta \leq 4 \pi$. In this case, the subgroup $\hat{M}(2)$ is a two element group comprising the center of $\hat{G}(2)$. Let $\mu_{0}$ and $\mu_{1}$ denote the trivial and nontrivial characters, respectively, of the subgroup $\hat{M}(2)$. Then the set of $\mu_{0}$-admissible classes $\Omega_{0}(\hat{K}(2))$, and the set of $\mu_{1}$-admissible classes $\Omega_{1}(\hat{K}(2))$ can be identified with the set of integers and with the set of half odd integers, respectively.

We write $d C_{i}=\operatorname{span}_{\mathbf{C}}\left\{e_{m}(\cdot): m \in \Omega_{i}(\hat{K}(2))\right\}$, for $i=0$ or 1 , and we write $L_{i}^{2}$ for the Hilbert space closure of $d C_{i}$ in $L^{2}(\hat{K}(2))$. Then the space $d C(\hat{K}(2))$ is linearly equivalent to an algebraic direct sum of the subspaces $d C_{0}$ and $d C_{1}$, and both of these subspaces are invariant under the action $d \Pi_{\Lambda}$ of $\mathbf{G}(2)$, for any character $\Lambda$. The Hilbert space $L^{2}(\hat{K}(2))$, in turn, is unitarily equivalent to a Hilbert space direct sum of the closed subspaces $L_{0}^{2}$ and $L_{1}^{2}$, and both of these subspaces are invariant under the group of operators $\Pi_{\Lambda}(\hat{G}(2))$, for any character $\Lambda$.

In statement (1) of Theorem 6 only condition (a) for irreducibility applies. This condition can be put in the following form: 
The $U(\mathbf{G}(2))$-module $\left[d C_{0}, d \Pi_{\Lambda}\right]$ is irreducible if and only if the character $\Lambda$ satisfies the condition $(\lambda+m)(\lambda-m) \neq 0$, for all integers $m$. The $U(\mathbf{G}(2))$ module $\left[d C_{1}, d \Pi_{\Lambda}\right]$ is irreducible if and only if $(\lambda+m)(\lambda-m) \neq 0$, for all half odd integers $m$.

According to Bargmann [1, Theorems 1 and 3], the following irreducible induced representations are unitarizable. (In case $n=2, \lambda_{0}=\lambda-\frac{1}{2}$.)

The $U(\mathbf{G}(2))$-module $\left[d C_{0}, d \Pi_{\Lambda}\right]$ is unitarizable for $\lambda_{0}$ imaginary (the principal series), and for real $\lambda_{0}$, with $-\frac{1}{2}<\lambda_{0}<\frac{1}{2}$. The $U(\mathbf{G}(2))$-module $\left[d C_{1}, d \Pi_{\Lambda}\right]$ is unitarizable for $\lambda_{0}$ imaginary and different from zero. It follows from our results, incidentally, and it is well known that the $U(\mathbf{G}(2))$-module $\left[d C_{1}, d \Pi_{\Lambda}\right]$ is not irreducible for $\lambda_{0}=0$.

The equivalence of the irreducible induced representation under the Weyl reflection $\lambda_{0} \rightarrow-\lambda_{0}$, follows from Theorem 6, statement (2). In the unitarizable cases mentioned above this equivalence is implicit in the results of Bargmann referred to. In fact, Bargmann's classification is in terms of the eigenvalues of the Casimir operator, namely, $\lambda^{2}-\lambda=\lambda_{0}^{2}-\frac{1}{4}$, which are invariant under the Weyl reflection.

We turn now to the nonirreducible situation. Assume that $s$ is an element of the set $\Omega_{i}(\hat{K}(2))$, for $i=0$ or 1 , and that $s<0$. Then in the notation of Theorem 6 , statement (4), $s^{\prime}=-s-1$. We assume that the character $\Lambda$ satisfies the condition $\lambda+s=0$, or equivalently, that the dual character $\Lambda^{\prime}=\Lambda P^{-1}$ satisfies the dual condition: $\lambda^{\prime}+s^{\prime}=0$. Then by specializing the statement (4) of Theorem 6 we have

$$
D_{\lambda 1}^{+} d C_{i}=\operatorname{span}_{\mathbf{C}}\left\{e_{m}(\cdot): m \geq-s\right\}, \quad D_{\lambda 1}^{-} d C_{i}=\operatorname{span}_{\mathbf{C}}\left\{e_{m}(\cdot): m \leq s\right\}
$$

and

$$
D_{\lambda^{\prime} 1}^{F} d C_{i}=\operatorname{span}_{\mathbf{C}}\left\{e_{m}(\cdot):-s^{\prime} \leq m \leq s^{\prime}\right\}
$$

The spaces $D_{\lambda^{\prime} 1}^{F} d C_{i}$ are finite dimensional, and invariant and irreducible under the group action $\Pi_{\Lambda^{\prime}}$, while the $U(\mathbf{G}(n))$-modules $\left[D_{\lambda 1}^{+} d C_{i}, d \Pi_{\Lambda}\right]$ and $\left[D_{\lambda 1}^{-} d C_{i}\right.$, $\left.d \Pi_{\Lambda}\right]$ are irreducible, by Theorem 6 .

The $\hat{G}(2)$-modules corresponding to the last two $U(\mathbf{G}(2))$-modules are unitariz able, according to Bargmann [1], and form the discrete series of unitary representations. We remark that as $s^{\prime}$ ranges over the nonnegative elements of $\Omega_{i}(R(2))$, and with $\lambda^{\prime}+s^{\prime}=0$, the modules $D_{\lambda^{\prime} 1}^{F} d C_{i}$ exhaust the finite-dimensional irreducible representations of $\hat{G}(2)$. This fact is implied by statement (5) of Theorem 6. It also follows directly by noting that the eigenvalues of the Casimir operator in these representations, namely $\lambda^{\prime 2}-\lambda^{\prime}=s^{\prime}\left(s^{\prime}+1\right)$, correspond uniquely to the finite-dimensional irreducible representations of $\hat{G}(2)$ with highest weight $s^{\prime}$. 
In the nonirreducible cases we have the following sum of mutually orthogonal projections: $D_{\lambda_{1}}^{+}+D_{\lambda_{1}}^{-}+D_{\lambda^{\prime} 1}^{F}=1$. We emphasize that although the representations $\left[D_{\lambda_{1}}^{ \pm} L_{i}^{2}, \Pi_{\Lambda}\right]$ are unitarizable, they are not unitarizable by the inner product on $L_{i}^{2}$, in general. An exception to this statement occurs when $i=1$ and $\lambda_{0}=0$, or equivalently, when $\lambda=\frac{1}{2}$. In this case we have $D_{\lambda^{\prime} 1}^{F}=0$, and the representations $\left[D_{\lambda_{1}}^{ \pm} L_{1}^{2}, \Pi_{\Lambda}\right]$ are supplementary subrepresentations of $\left[L_{1}^{2}, \Pi_{\Lambda}\right]$. This is the single nonirreducible case of the principal series. We note that the finitedimensional representations occur as subrepresentations of induced representations corresponding to the character $-\lambda_{0}$, while the supplementary discrete series occurs as a subrepresentation of the induced representation corresponding to the parameter $\lambda_{0}$. This fact is a folk theorem, and was recently pointed out explicitly by Sally [16].

Finally, we observe that the infinitesimal equivalence of quotient representations and subrepresentations given in statement (4) of Theorem 6 reduce to the following statements in the nonirreducible situation. We recall that for $s$ $\in \Omega_{i}(\hat{K}(2))$ and $s<0$, we have $\lambda+s=0, \lambda^{\prime}-(1+s)=0, \lambda=d \Lambda(H)$, and $\lambda^{\prime}=d \Lambda^{\prime}(H)$.

$$
\begin{aligned}
{\left[D_{\lambda^{\prime} 1}^{F} d C_{i}, d \Pi_{\Lambda^{\prime}}\right] } & \cong\left[d C_{i}, d \Pi_{\Lambda}\right] /\left[\left(D_{\lambda 1}^{-}+D_{\lambda 1}^{+}\right) d C_{i}, d \Pi_{\Lambda}\right], \\
{\left[D_{\lambda 1}^{-} d C_{i}, d \Pi_{\Lambda}\right]+\left[D_{\lambda_{1}}^{+} d C_{i}, d \Pi_{\Lambda}\right] } & \cong\left[d C_{i}, d \Pi_{\Lambda^{\prime}}\right] /\left[D_{\lambda^{\prime} 1}^{F} d C_{i}, d \Pi_{\Lambda^{\prime}}\right] .
\end{aligned}
$$

In this case, these isomorphisms can be obtained by elementary methods, but we will not pursue this point here.

B. The case $n=3$. In this case $\hat{G}(3)$ is isomorphic to the group $\operatorname{SL}(2, \mathbf{C})$, and also the universal covering group of the identity component of the standard homogeneous Lorentz group. The subgroup $\hat{K}(3)$ is isomorphic to $\operatorname{Spin}(3)$ and $\mathrm{SU}(2)$. We recall that the symbol $\widehat{\exp }$ denotes the exponential map of $\hat{G}(3)$ into $\operatorname{Spin}(1,3)$. The subgroup $\hat{M}(3)$ is the circle group given by $\left\{\widehat{\exp }\left(\Theta X_{23}\right)\right.$ : $0 \leq \Theta \leq 4 \pi\}$. Thus, the irreducible representations of this subgroup are parameterized by half integers corresponding to the characters of this abelian group. We note that the Lie subalgebra $\mathbf{M}(3)$ is a Cartan subalgebra of $\mathbf{K}(3)$. The irreducible representations of $\mathscr{K}(3)$ are parameterized by the highest weights $\ell \varepsilon_{1}$, with $2 \ell$ a nonnegative integer. The irreducible representation of $\hat{K}(3)$ corresponding to the highest weight $\ell \varepsilon_{1}$ has dimension $2 l+1$. We identify the classes of irreducible unitary representations of compact groups with their highest weights. Thus,

$$
\Omega(\hat{M}(2))=\left\{m \varepsilon_{1}: 2 m \in \mathbf{Z}\right\},
$$

and for $m \varepsilon_{1} \subseteq \Omega(\hat{M}(2))$, the corresponding $m$-admissible classes comprise the set:

$$
\Omega_{m}(\hat{K}(3))=\left\{\ell \varepsilon_{1}: \ell=|m|+n, n=0,1,2,3, \ldots\right\} .
$$

This fact is a special case of inequality (7b) in Lemma 4. 
Let $m \varepsilon_{1} \in \Omega(\hat{M}(3))$. Then the spaces $L_{\mu}^{2}(\hat{K}(n), H)$ and $d C_{\mu}(\hat{K}(n), H)$ specialize, in this case, to the spaces $L_{m}^{2}$ and $d C_{m}$ respectively, where $L_{m}^{2}$ denotes the space of square-integrable function classes on $K(3)$ which satisfy the condition

$$
F\left(\exp \left(\Theta X_{23}\right) k\right)=\exp \left[m\left((-1)^{1 / 2} \Theta\right)\right] F(k),
$$

with $k \in \hat{K}(3), F \in L_{m}^{2}$, and $d C_{m}$ is the corresponding dense subspace consisting of $\hat{R}$ (3)-finite functions. We remark that in case $m=0$, the space $d C_{m}$ reduces to the algebra $\delta$ used in the proof of Theorem 2 , which in this case reduces to a set of finite linear combinations of the classical spherical harmonics on the 2-sphere.

From Theorem 6, statement (1), the necessary and sufficient condition that the $U(\mathbf{G}(3))$-module $\left[d C_{m}, d \Pi_{\Lambda}\right]$ be irreducible, or equivalently, that the $\hat{G}(3)$-module $\left[L_{m}^{2}, \Pi_{\Lambda}\right]$ be topologically irreducible, is that, for all $\ell \varepsilon_{1} \in \Omega_{m}(R(3)), \lambda+\ell \neq 0$, and $\lambda-\ell-1 \neq 0$, or if $\lambda-\ell-1=0$, then $\ell=|m|$. This condition may be rewritten in terms of the inducing parameter as follows: (In this case $\lambda_{0}$ $=\lambda-(n-1) / 2=\lambda-1$.)

$$
\left(\lambda_{0}+\ell+1\right)\left(\lambda_{0}-\ell-1\right) \neq 0
$$

for all $\ell \varepsilon_{1} \in \Omega_{m}(\hat{K}(3))$. This condition agrees with the one given in Naimark [12, Theorem 16, p. 295]. The statement in that theorem relating to the nonirreducible situation is inaccurate, however. (See the discussion below.) We remark that the parameter $\lambda_{0}$ corresponds to the parameter $(-1)^{1 / 2} \rho / 2$ in the statement of that theorem, and the parameter $m$ used here corresponds to the parameter $m / 2$ in the statement of the theorem of Naimark cited above.

We note that if $m \varepsilon_{1}$ is an element of $\Omega(\hat{M}(3))$, then $-m \varepsilon_{1}$ corresponds to the complex conjugate representation of $\hat{M}(3)$. Hence, by statement (2) of Theorem 6 , the induced representation corresponding to the parameters $\left(-m,-\lambda_{0}\right)$ is infinitesimally equivalent to the induced representation corresponding to the parameters $\left(m, \lambda_{0}\right)$. This fact is also stated in the theorem of Naimark cited above.

The unitary representations of $\hat{G}(3)$ were classified independently by Bargmann and by Gelfand and Naimark. A complete discussion of the unitary representations is contained in the book by Naimark [12]. The unitarizable representations are the following ones. For $m \varepsilon_{1} \in \Omega(\hat{M}(3))$, and for $\lambda_{0}$ imaginary, the representation $\left[L_{m}^{2}, \Pi_{\Lambda}\right]$ is unitary. This is the case of the principal series; they are irreducible according to the condition stated above. When $m=0$ and when $\lambda_{0}$ is real with $\lambda_{0} \neq 0,-1 \leq \lambda_{0} \leq 1$, the induced representations are unitarizable, corresponding to the complementary series, with $\lambda_{0}$ and $-\lambda_{0}$ yielding infinitesimally equivalent, and hence, unitarily equivalent representations. We remark that the identity representation occurs as a subrepresentation of the induced representation in this case when $\lambda_{0}=1$. All other unitary representations are, by the remarks made above, infinitesimally equivalent to the above irreducible induced representations. 
We turn now to the nonirreducible situation. Let $s \varepsilon_{1} \in \Omega_{m}(\hat{K}(3))$. According to Theorem 6 , statement (1), this situation occurs when either $\lambda+s=0$, or when $\lambda-s-1=0$ and $s+1 \neq m$. As in statement (3) of that theorem it is only necessary to assume that $\lambda+s=0$, since the Weyl reflection $\lambda^{\prime}=-\lambda+2$ satisfies the second condition with $s$ replaced by $s+1$. The sets $D_{\lambda}^{+}$and $D_{\lambda^{\prime}}^{-}$in that statement specialize to the following ones:

$$
\begin{aligned}
& D_{\lambda}^{+}=\left\{\ell \varepsilon_{1}: \ell=s+1, s+2, s+3, \ldots\right\}, \\
& D_{\lambda^{-}}^{-}=\left\{\ell \varepsilon_{1}: \ell=s, s-1, \ldots,|m|+1,|m|\right\} .
\end{aligned}
$$

We note that the set $D_{\lambda^{\prime}}^{-}$is finite. Let $D_{\lambda}^{+}$and $D_{\lambda^{\prime}}^{-}$be the projections corresponding to the sets $D_{\lambda}^{+}$and $D_{\lambda^{\prime}}^{-}$respectively, as in Theorem 6. By that theorem, the representations $\left[D_{\lambda}^{+} d C_{m}, d \Pi_{\Lambda}\right]$ and $\left[D_{\lambda^{\prime}}^{-} d C_{m}, d \Pi_{\Lambda}\right]$ are irreducible, the latter being finite dimensional, where we recall that $\Lambda^{\prime}=\Lambda^{-1} P$, or infinitesimally, $\lambda^{\prime}=-\lambda+2$. The module isomorphisms of statement (3e) reduce to the following ones:

$$
\begin{aligned}
{\left[D_{\lambda}^{+} d C_{m}, d \Pi_{\Lambda}\right] } & \cong\left[d C_{-m}, d \Pi_{\Lambda^{\prime}}\right] /\left[D_{\lambda^{\prime}}^{-} d C_{-m}, d \Pi_{\Lambda^{\prime}}\right] \\
{\left[D_{\lambda^{\prime}}^{-} d C_{m}, d \Pi_{\Lambda^{\prime}}\right] } & \cong\left[d C_{-m}, d \Pi_{\Lambda}\right] /\left[D_{\lambda}^{+} d C_{-m}, d \Pi_{\Lambda}\right]
\end{aligned}
$$

Note that in this case there is a nonunitarizable discrete series of representations which bear the same relationship to the finite-dimensional ones as in the case of $\operatorname{SL}(2, \mathbf{R})$. This fact was ignored in the results of Naimark cited above.

C. The cases $n \geq 4$. The case $n=4$ corresponds to the deSitter group, and $\hat{G}(4)$ is its universal covering group. A complete classification of the unitary representations of this group can be found in Dixmier [6]. The methods used by Dixmier are algebraic, and are a refinement of those of the physicist Thomas. (The references are given in [6].) Dixmier uses those results of Harish-Chandra, cited in the introduction, which give a one-to-one correspondence between unitary equivalence classes of unitary irreducible representations and certain infinitesimal equivalence classes of irreducible quasi-simple representations. Dixmier then proceeds "from scratch" to analyze the irreducible $U(\mathbf{G}(4))$-modules that correspond to the irreducible unitary representations. A global description, in terms of induced representations, of Dixmier's representations is given by Takahashi [15]. Some of Dixmier's results are generalized by Takahashi to general $n$.

We will now list all the irreducible quasi-simple representations for $\hat{G}(4)$ as determined by Theorem 6, and point out which of these correspond to the unitary representations of Dixmier.

In case $n=4$, the group $\hat{K}(4)$ is isomorphic to the direct product of two subgroups each isomorphic to Spin(3). Dixmier takes advantage of this splitting by expressing the highest weights of $\mathbf{K}(4)$ in terms of the roots $\varepsilon_{1}+\varepsilon_{2}, \varepsilon_{1}-\varepsilon_{2}$. Let $\Lambda_{\omega}$ be a highest weight of $\mathbf{K}(4)$, and let $k$ and $k^{\prime}$ be the components of $\Lambda_{\omega}$ 
with respect to $\varepsilon_{1}+\varepsilon_{2}$ and $\varepsilon_{1}-\varepsilon_{2}$ respectively. Then $k$ and $k^{\prime}$ are half integers and

$$
\Lambda_{\omega}=\Lambda_{\omega_{1}} \varepsilon_{1}+\Lambda_{\omega_{2}} \varepsilon_{2}=k\left(\varepsilon_{1}+\varepsilon_{2}\right)+k^{\prime}\left(\varepsilon_{1}-\varepsilon_{2}\right) .
$$

Note also that in this case the subgroup $\hat{M}(4)$ is isomorphic to $\operatorname{Spin}(3)$, and the Lie algebra $\mathbf{M}(4)=\operatorname{span}_{\mathbf{R}}\left\{X_{23}, X_{24}, X_{34}\right\}$. Thus, again identifying unitary equivalence classes of irreducible unitary representations of compact groups and their highest weights, we have

$$
\Omega(\hat{M}(4))=\left\{m \varepsilon_{2}: 2 m=0,1,2,3, \ldots\right\}
$$

By the inequalities (7a) of Lemma 4 , for each $m \varepsilon_{2} \in \Omega(\hat{M}(4))$, the $m$-admissible classes are given by

$$
\begin{aligned}
\Omega_{m}(\hat{K}(4))= & \left\{k\left(\varepsilon_{1}+\varepsilon_{2}\right)+k^{\prime}\left(\varepsilon_{1}-\varepsilon_{2}\right):\right. \\
& \left.k+k^{\prime}=m, m+1, m+2, \ldots ; k-k^{\prime}=m, m-1, \ldots,-m\right\} .
\end{aligned}
$$

The conditions for irreducibility given in statement (1) of Theorem 6 specialize as follows. By setting $i=1$ in that statement, we must have

(I) $\lambda+\left(k+k^{\prime}\right) \neq 0$, and $\lambda-\left(k+k^{\prime}\right)-2 \neq 0$, except possibly when $k+k^{\prime}$ $=m$.

By setting $i=2$ in that statement, we must have

(II) $\lambda-\left(k-k^{\prime}\right)-1 \neq 0$, and $\lambda+\left(k-k^{\prime}\right)-1 \neq 0$, except possibly when $\left(k-k^{\prime}\right)=m$.

In the important special case when $m=0$, the class one representations, we always have $\left(k-k^{\prime}\right)=0=m$. Hence, for class one representations, only condition (I) applies.

While discussing the $\hat{K}(4)$-module structure of the irreducible unitary representations, Dixmier introduces a parameter $p$, which is defined as the minimum value of $k+k^{\prime}$ occuring in the given representation. For the irreducible case this minimum value is the minimum value of the quantity $k+k^{\prime}$ in the set of $m$ admissible classes, which is precisely $m$. Hence, the $R(4)$-module structure for the irreducible cases corresponds to Figure 2 in the paper of Dixmier. To decide which of the irreducible cases are unitarizable, according to Dixmier, we examine the eigenvalues of the Casimir operator. By Lemma 6, the latter are given by

$$
d \Pi_{\Lambda \mu}(\Omega)=\lambda^{2}-3 \lambda+m(m+1)=\lambda_{0}^{2}-9 / 4+m(m+1),
$$

where $\lambda_{0}$ is the inducing parameter given by $\lambda=\lambda_{0}+(n-1) / 2=\lambda_{0}+3 / 2$. In terms of a parameter $\sigma$ introduced by Dixmier, these eigenvalues are

$$
d \Pi_{\Lambda \mu}(\Omega)=-2-\sigma+m(m+1) .
$$


In order that the irreducible representations be unitarizable, one must have

(a) For $m=1 / 2,3 / 2,5 / 2, \ldots, \sigma>1 / 4$, or $\lambda_{0}^{2}<0$.

(b) For $m=1,2,3, \ldots, \sigma>0$, or $\lambda_{0}^{2}<1 / 4$.

(c) For $m=0, \sigma>-2$, or $\lambda_{0}^{2}<9 / 4$.

We remark that by statement (2) of Theorem 6 , the induced representations corresponding to the parameters $\left(m, \lambda_{0}\right)$ and $\left(m,-\lambda_{0}\right)$ are infinitesimally equivalent, and that these are the only equivalences. In case these parameters satisfy conditions (a), (b), and (c) above, $\left(m, \lambda_{0}\right)$ and $\left(m,-\lambda_{0}\right)$ also correspond to unitarily equivalent representations, by the theorem of Harish-Chandra quoted in the introduction. This equivalence of induced representations does not appear in Dixmier's work, since his classification is based essentially on the eigenvalues of the Casimir operator and the $\hat{K}(4)$-module structure of the possible $U(G(4))$ modules.

Note that the parameters which satisfy conditions (a), (b), and (c) above indeed correspond to the irreducible case. In fact, for condition (c) we have, for all $\left(k+k^{\prime}\right) \geq m=0$,

$$
0 \neq \lambda_{0}^{2}-\left[3 / 2+\left(k+k^{\prime}\right)\right]^{2}=\left(\lambda-\left(k+k^{\prime}\right)-3\right)\left(\lambda+\left(k+k^{\prime}\right)\right) .
$$

Hence, irreducibility follows by the remark made concerning class one representations. Moreover, if $\left(m, \lambda_{0}\right)$ satisfies conditions (a) and (b) above, we must have, for all $k+k^{\prime} \geq m$ and $k-k^{\prime}$ with $m \leq k-k^{\prime} \leq-m$,

$$
0 \neq \lambda_{0}^{2}-\left[3 / 2+\left(k+k^{\prime}\right)\right]^{2}=\left(\lambda-\left(k+k^{\prime}\right)-3\right)\left(\lambda+\left(k+k^{\prime}\right)\right),
$$

and

$$
0 \neq \lambda_{0}^{2}-\left[1 / 2+\left(k+k^{\prime}\right)\right]^{2}=\left(\lambda-\left(k+k^{\prime}\right)-1\right)\left(\lambda+\left(k-k^{\prime}\right)+1\right) .
$$

Hence, irreducibility follows by statements (I) and (II) above.

If $\lambda_{0}^{2}<0$, then $\lambda_{0}$ is purely imaginary. This situation corresponds to the principal series of unitary representations. In this case the induced representations $\left[L_{\mu}^{2}(R(4)), \Pi_{\Lambda}\right]$ are unitary, where $\mu=\varepsilon_{2} m$. In case (c) we also have the possibility of $\lambda_{0}$ being real with $-3 / 2<\lambda_{0}<3 / 2$. This is the case of the class one complementary series. An explicit construction of the appropriate inner product for this case is given by Takahashi [17], for general $n$. In case (b) we also have the possibility of $\lambda_{0}$ real with $-\frac{1}{2}<\lambda_{0}<\frac{1}{2}$. This case corresponds to another complementary series. A global description of these and more general complementary series is given in the case of general $n$ by Knapp and Stein [15].

We turn now to the nonirreducible cases for $n=4$. First, we examine the consequences of statement (4) of Theorem 6. Since $p=2$ here, we assume that $\mu=m \varepsilon_{1} \in \Omega(\hat{M}(4))$, and that there exists an $m$-admissible class $\Lambda_{\omega}$ such that $s=\Lambda_{\omega_{2}}=k-k^{\prime}$ satisfies the equation $\lambda+s-1=0$. For the dual character $\Lambda^{\prime}$ we have $\lambda^{\prime}=-\lambda+3$, and we have $\lambda^{\prime}+s^{\prime}-1=0$, where $s^{\prime}=-(s+1)$. The sets $D_{\lambda 2}^{+}, D_{\lambda 2}^{-}$, and $D_{\lambda^{\prime} 2}^{F}$ of Theorem 5 reduce in this case to the following ones: 


$$
\begin{aligned}
D_{\lambda 2}^{+}= & \left\{k\left(\varepsilon_{1}+\varepsilon_{2}\right)+k^{\prime}\left(\varepsilon_{1}-\varepsilon_{2}\right):\right. \\
& \left.k+k^{\prime}=m, m+1, m+2, \ldots ; k-k^{\prime}=m, m-1, \ldots,-s\right\}, \\
D_{\lambda 2}^{-}= & \left\{k\left(\varepsilon_{1}+\varepsilon_{2}\right)+k^{\prime}\left(\varepsilon_{1}-\varepsilon_{2}\right):\right. \\
& \left.k+k^{\prime}=m, m+1, m+2, \ldots ; k-k^{\prime}=-m,-m+1, \ldots, s\right\}, \\
D_{\lambda^{\prime} 2}^{F}= & \left\{k\left(\varepsilon_{1}+\varepsilon_{2}\right)+k^{\prime}\left(\varepsilon_{1}-\varepsilon_{2}\right):\right. \\
& \left.k+k^{\prime}=m, m+1, m+2, \ldots ; k-k^{\prime}=s^{\prime}, s^{\prime}-1, \ldots,-s^{\prime}\right\} .
\end{aligned}
$$

From Lemma 6 the Casimir operator for this case is, with $\lambda=d \Lambda(H)$,

$$
d \Pi_{\Lambda}(\Omega)=-2+s+s^{2}+m(m+1)=-2+s^{\prime}+s^{2}+m(m+1) .
$$

The $\hat{K}(4)$-module structure represented by the sets $D_{\lambda 2}^{+}$and $D_{\lambda 2}^{-}$correspond to Figures 4 and 3 of [6], respectively, with Dixmier's parameter $q$ equal to $-s$. By comparing the eigenvalues of the Casimir operator above with those of Dixmier, and by comparing the $K(4)$-module structures, we get the following results:

The group representations corresponding to the $U(\mathbf{G}(4))$-module $\left[D_{\lambda 2}^{\mp} d C_{\mu}\right.$, $\left.d \Pi_{\Lambda}\right]$ are unitarizable, and correspond to Dixmier's representations $\Pi_{p, q}^{ \pm}$, with $p=m, q=-s$. The group representations corresponding to the $U(\mathbf{G}(4))$-module $\left[D_{\lambda 2}^{F} d C_{\mu}, d \Pi_{\Lambda^{\prime}}\right]$ is not unitarizable, in general, except when $s^{\prime}=0$. In that case, the latter representation corresponds to the representation $\Pi_{p 0}$ of Dixmier, with $p=m$, and the corresponding $\hat{K}(4)$-module structure is that exhibited in Figure 5 of Dixmier's paper.

When $s^{\prime}$ is not necessarily equal to zero, the representations $\left[D_{\lambda 2}^{F} d C_{\mu}, d \Pi_{\Lambda}\right]$ has a relationship to the unitarizable discrete series as do the finite-dimensional representations in the case of SL $(2, \mathbf{R})$. In particular, the discrete series occur in pairs which are mutually orthogonal relative to the inner product on $L_{\mu}^{2}(R(4))$, and we have the $U(\mathbf{G}(4))$-module isomorphisms as given in statement (4) of Theorem 6.

Next, we discuss the consequence of statement (3) of Theorem 6. Here one must assume that $i=1$. Again, $\mu=m \varepsilon_{2} \in \Omega(\hat{M}(4))$. Assume the existence of a $\mu$-admissible class $[\omega]$ such that $\Lambda_{\omega_{1}}=s$, and $\lambda+s-3=0$. For $n=4$ the sets $D_{\lambda}^{+}$and $D_{\lambda}^{-}$specialize to the following:

$$
\begin{aligned}
D_{\lambda}^{+}= & \left\{k\left(\varepsilon_{1}+\varepsilon_{2}\right)+k^{\prime}\left(\varepsilon_{1}-\varepsilon_{2}\right):\right. \\
& \left.k+k^{\prime}=s+1, s+2, \ldots ; k-k^{\prime}=m, m-1, \ldots,-m\right\}, \\
D_{\lambda^{\prime}}^{-}= & \left\{k\left(\varepsilon_{1}+\varepsilon_{2}\right)+k^{\prime}\left(\varepsilon_{1}-\varepsilon_{2}\right):\right. \\
& \left.k+k^{\prime}=s, s-1, \ldots, m ; k-k^{\prime}=m, m-1, \ldots,-m\right\} .
\end{aligned}
$$

Note that $D_{\lambda^{\prime}}^{-}$is a finite set; it yields the $\hat{K}(4)$-module structure of the irreducible finite-dimensional representation with highest weight $(s, m)$. Neither of the 
corresponding $U(\mathbf{G}(4))$-modules, $\left[D_{\lambda}^{+} L_{\mu}^{2}(\hat{K}(4)), \Pi_{\Lambda}\right]$ and $\left[D_{\lambda^{\prime}}^{-} L_{\mu}^{2}(\hat{K}(4)), \Pi_{\Lambda^{\prime}}\right]$, is unitarizable, in general. By statement $(3 \mathrm{e})$ of Theorem 6 , one is infinitesimally equivalent to a quotient of the other.

We conclude with some remarks about the class one representations of $\hat{G}(n)$ for general $n$, that is, representations whose restriction to $\hat{K}(n)$ contain the identity representation. This situation occurs when $\mu$ is the identity representation. Hence, in particular, such an induced representation must be an ordinary representation, that is, a representation of $G(n)$ as well as of $\hat{G}(n)$. In the case of general semisimple matrix groups, Kostant [14] gives general criteria for the nonirreducibility of the induced representations under study. For the groups under study here, his Theorem 2 yields the following criteria for nonirreducibility: $|\lambda-(n-1) / 2| \geq(n-1) / 2$, and $\lambda \equiv 0 \bmod \mathbf{Z}$. In comparing this result with statement (1) of Theorem 6 , we note that for $i>1$ we have $\Lambda_{\omega i}=\Lambda_{\mu i-1}=0$ $=\Lambda_{\mu i}$. Hence, the nonirreducibility criterion in this case obtains from statement (la) with $i=1$, and agrees with the result deduced from Kostant's theorem.

\section{REFERENCES}

1. V. Bargmann, Irreducible unitary representations of the Lorentz group, Ann. of Math. (2) 48 (1947), 568-640. MR $9,133$.

2. H. Boerner, Darstellungen von Gruppen. Mit Berücksichtigung der Bedürfnisse der modernen Physik, Zweite, überarbeitete Auflage, Die Grundlehren der math. Wissenschaften, Band 74, Springer-Verlag, Berlin and New York, 1967. MR 37 \# 5307.

3. R. Brauer, Sur la multiplication des charactéristiques des groupes continus et semisimples, C. R. Acad. Sci. Paris 204 (1937), 1784-1786.

4. F. Bruhat, Sur les représentations induites des groupes de Lie, Bull. Soc. Math. France 84 (1956), 97-205. MR 18, 907.

5. C. Chevalley, Theory of Lie groups. I, Princeton Math. Series, vol. 8, Princeton Univ. Press, Princeton, N.J., 1946. MR 7, 412.

6. J. Dixmier, Représentations intégrables du groupe de De Sitter, Bull. Soc. Mạth. France 89 (1961), 9-41. MR 25 \#4031.

7. J. M. G. Fell, Non-unitary dual spaces of groups, Acta Math. 114 (1965), 267-310. MR 32 \#4210.

8. R. Godement, $A$ theory of spherical functions. I, Trans. Amer. Math. Soc. 73 (1952), 496-556. MR 14, 620 .

9a. Harish-Chandra, Representations of a semisimple Lie group on a Banach space. I, Trans. Amer. Math. Soc. 75 (1953), 185-243. MR 15, 100.

9b.—-, Representations of a semisimple Lie group. II, Trans. Amer. Math. Soc. 76 (1954), $26-65$. MR 15, 398.

9c.—, Representations of a semisimple Lie group. III, Trans. Amer. Math. Soc. 76 (1954), 234-253. MR 16, 11.

9d._- The Plancherel formula for complex semisimple Lie groups, Trans. Amer. Math. Soc. 76 (1954), 485-528. MR 16, 111.

10. S. Helgason, Differential geometry and symmetric spaces, Pure and Appl. Math., vol. 12, Academic Press, New York, 1962. MR 26 \#2986.

11a. T. Hirai, On infinitesimal operators of irreducible representations of the Lorentz group of $n$-th order, Proc. Japan Acad. 38 (1962), 83-87. MR 25 \#2146.

$11 \mathrm{~b} . \longrightarrow$, On irreducible representations of the Lorentz group of $n$-th order, Proc. Japan Acad. 38 (1962), 258-262. MR 32 \#8844. 
11c. T. Hirai, The characters of irreducible representations of the Lorentz group of $n$-th order, Proc. Japan Acad. 41 (1965), 526-531. MR 33 \#185.

12. M. A. Naìmark, Linear representations of the Lorentz group, Fizmatgiz, Moscow, 1958; English transl., Macmillan, New York, 1964. MR 21 \#4995; MR 30 \#1211.

13. N. Jacobson, Lie algebras, Interscience Tracts in Pure and Appl. Math., no. 10, Interscience, New York, 1962. MR 26 \#1345.

14. B. Kostant, On the existence and irreducibility of certain series of representations, Bull. Amer. Math. Soc. 75 (1969), 627-642. MR 39 \#7031.

15. A. W. Knapp and E. M. Stein, Intertwining operators for semisimple groups, Ann. of Math. (2) 93 (1971), $489-578$.

16. P. J. Sally, Jr., Intertwining operators and the representations of $\operatorname{SL}(2, \mathbf{R})$, J. Functional Analysis 6 (1970), 441-453.

17. R. Takahashi, Sur les représentations unitaires des groupes de Lorentz généralisés, Bull. Soc. Math. France 91 (1963), 289-433.

18. E. Thieleker, On the irreducibility of nonunitary induced representations of certain semidirect products, Trans. Amer. Math. Soc. 164 (1972), 353-369.

19. A. Weil, L'intégration dans les groupes topologiques et ses applications, 2ième éd., Actualités Sci. Indust., no. 869, Hermann, Paris, 1951. MR 3, 198.

Department of Mathematics, University of South Florida, Tampa, Florida 33620 